\title{
The transfer of resonance line polarization with partial frequency redistribution and $J$-state interference
}

\section{Theoretical approach and numerical methods}

\author{
L. Belluzzi ${ }^{1,2}$ and J. Trujillo Bueno ${ }^{1,2,3}$ \\ ${ }^{1}$ Instituto de Astrofísica de Canarias, 38205 La Laguna, Tenerife, Spain \\ e-mail: belluzzi@iac.es \\ 2 Departamento de Astrofísica, Facultad de Física, Universidad de La Laguna, 38206 La Laguna, Tenerife, Spain \\ 3 Consejo Superior de Investigaciones Científicas, 28006 Madrid, Spain
}

Received 29 March 2013 / Accepted 19 December 2013

\section{ABSTRACT}

\begin{abstract}
The linear polarization signals produced by scattering processes in strong resonance lines are rich in information on the magnetic and thermal structure of the chromosphere and transition region of the Sun and of other stars. A correct modeling of these signals requires accounting for partial frequency redistribution effects, as well as for the impact of quantum interference between different fine structure levels ( $J$-state interference). In this paper, we present a theoretical approach suitable for modeling the transfer of resonance line polarization when taking these effects into account, along with an accurate numerical method of solution of the problem's equations. We consider a two-term atom with unpolarized lower term and infinitely sharp lower levels, in the absence of magnetic fields. We show that by making simple formal substitutions on the quantum numbers, the theoretical approach derived here for a two-term atom can also be applied to describe a two-level atom with hyperfine structure. An illustrative application to the Mg II doublet around $2800 \AA$ is presented.
\end{abstract}

Key words. atomic processes - line: formation - polarization - radiative transfer - scattering - stars: atmospheres

\section{Introduction}

To probe the thermal, dynamic and magnetic properties of the chromosphere and transition region of the Sun and of other stars, we need to measure and interpret the intensity and polarization profiles of strong resonance lines, such as $\mathrm{Mg}$ II $\mathrm{h} \& \mathrm{k}$, hydrogen Ly- $\alpha$, or He II $304 \AA$ A. Interpretation of the Stokes parameters of the observed radiation requires solving a non-LTE radiative transfer problem that can be very complex, especially when the main interest lies in modeling the spectral details of the linear polarization signals produced by scattering processes and their modification by the Hanle effect. One of the main difficulties occurs because scattering is a second-order process within the framework of quantum electrodynamics (e.g., Casini \& Landi Degl'Innocenti 2007), where frequency correlations between the incoming and outgoing photons can occur (partial frequency redistribution, PRD). Another aspect contributing to the complexity of the problem is the need to account for the impact of quantum interference (or coherence) between pairs of magnetic sublevels pertaining either to the same $J$-level ( $J$ being the level's total angular momentum) or to different $J$-levels of the same term ( $J$-state interference; see Belluzzi \& Trujillo Bueno 2011, and references therein). An additional complication stems from the fact that the plasma of a stellar chromosphere can be highly inhomogeneous and dynamic, which implies the need to solve the non-equilibrium problem of the generation and transfer of polarized radiation in realistic three-dimensional stellar atmospheric models (e.g., Štěpán \& Trujillo Bueno 2013).

As shown by Belluzzi \& Trujillo Bueno (2012), the joint action of PRD and $J$-state interference produces a complex scattering polarization $Q / I$ profile across the $\mathrm{h}$ and $\mathrm{k}$ lines of $\mathrm{Mg}$ II, with large amplitudes in their wings. The same happens with the Ly- $\alpha$ lines of hydrogen and ionized helium (Belluzzi et al. 2012). In these Letters we only had room to briefly outline our approach to the problem and to present the main results. The aim of this paper is therefore to describe the theoretical framework and the numerical methods we have developed for modeling the transfer of resonance line polarization taking PRD and $J$-state interference into account.

An atomic model accounting for the presence of quantum interference between pairs of sublevels pertaining either to the same $J$-level or to different $J$-levels within the same term is the multiterm model atom described in the monograph by Landi Degl'Innocenti \& Landolfi (2004; hereafter LL04). While a quantum-mechanical PRD theory for a two-level atom is available (see Bommier 1997a,b), a self-consistent PRD theory for multiterm systems has not been derived yet. The approximate approach that we propose in this paper is heuristically built starting from two distinct theoretical frameworks: the polarization theory presented in LL04 and the theoretical scheme described in Landi Degl'Innocenti et al. (1997).

The density matrix theory of LL04 is based on a lowest order perturbative expansion of the atom-photon interaction within the framework of quantum electrodynamics. Within this theoretical approach, a scattering event is described as a temporal succession of independent first-order absorption and re-emission processes, which is justified when the plasma conditions are such that the coherency of scattering is completely destroyed (limit of complete redistribution in frequency, CRD).

The theoretical approach developed by Landi Degl'Innocenti et al. (1997) allows us to describe the opposite limit of purely 
coherent scattering in the atom rest frame. This theory is based on the assumption that the atomic energy levels are composed by a continuous distribution of so-called metalevels (see, Hubeny et al. 1983a,b). In Landi Degl'Innocenti et al. (1997), the expression of the redistribution matrix for coherent scattering $\left(R_{\mathrm{II}}\right.$, following the terminology introduced by Hummer 1962) is derived for different atomic models. In particular, the authors consider the case of a two-term atom, under the assumptions that the fine structure levels of the lower term are infinitely sharp, and that the magnetic sublevels of the lower term are evenly populated and no interference is present between them (unpolarized lower term).

We consider a two-term model atom with unpolarized lower term and infinitely sharp lower levels. We point out that this atomic model is not just of academic interest, but it is perfectly suitable for investigating several resonance doublets of high diagnostic interest, such as the $\mathrm{h} \& \mathrm{k}$ lines of Mg II, or the Ly- $\alpha$ lines of hydrogen and ionized helium. This is because the lower level of these lines has $J=1 / 2$ (it cannot be polarized, unless the incident radiation has net circular polarization) and it is the ground level (its long lifetime justifies the infinitely-sharp level assumption). By analogy with the two-level atom case (briefly recalled in Sect. 2), we assume that in the atom rest frame the total redistribution matrix for such a two-term atom is given by a linear combination of two terms, one describing the limit of coherent scattering $\left(R_{\mathrm{II}}\right)$ and one describing the CRD limit $\left(R_{\mathrm{III}}\right)$. Starting from the two-term atom theory presented in LL04, as generalized by Belluzzi et al. (2013) for including the effect of inelastic and superelastic collisions ${ }^{1}$, we derive an approximate expression of the $R_{\mathrm{III}}$ redistribution matrix, under the assumption of CRD in the observer's frame (Sect. 3.1). Concerning the $R_{\text {II }}$ redistribution matrix, we start from the expression derived by Landi Degl'Innocenti et al. (1997) in the atomic rest frame. We derive the corresponding expressions in the observer's frame taking Doppler redistribution into account, and we include the effect of inelastic and superelastic collisions by analogy with the case of $R_{\text {III }}$ (Sect. 3.2). The formulation of the radiative transfer problem is presented in Sect. 5, while the iterative method for the solution of the non-LTE problem is discussed in Sect. 6. An illustrative application of our modeling scheme is shown in Sect. 7.

\section{Redistribution matrix for a two-level atom}

A quantum mechanical derivation of the redistribution matrix for polarized radiation, for a two-level atom with unpolarized and infinitely sharp lower level has been carried out by Domke \& Hubeny (1988) and Bommier (1997a,b). Following the convention according to which primed quantities refer to the incoming photon and unprimed quantities to the scattered photon, in the atom rest frame this redistribution matrix can be written in the form (see Eq. (109) of Bommier 1997a)

$$
\begin{aligned}
R_{i j}\left(\xi^{\prime}, \boldsymbol{\Omega}^{\prime} ; \xi, \boldsymbol{\Omega}\right)=\sum_{K=0}^{2} W_{K}\left(J_{\ell}, J_{u}\right)\left[P^{(K)}\left(\mathbf{\Omega}^{\prime}, \boldsymbol{\Omega}\right)\right]_{i j} \\
\times\left[\frac{\Gamma_{\mathrm{R}}}{\Gamma_{\mathrm{R}}+\Gamma_{\mathrm{I}}+\Gamma_{\mathrm{E}}} \delta\left(\xi-\xi^{\prime}\right) \phi\left(v_{0}-\xi\right)\right. \\
\left.\quad+\frac{\Gamma_{\mathrm{R}}}{\Gamma_{\mathrm{R}}+\Gamma_{\mathrm{I}}+D^{(K)}} \frac{\Gamma_{\mathrm{E}}-D^{(K)}}{\Gamma_{\mathrm{R}}+\Gamma_{\mathrm{I}}+\Gamma_{\mathrm{E}}} \phi\left(v_{0}-\xi^{\prime}\right) \phi\left(v_{0}-\xi\right)\right],
\end{aligned}
$$

\footnotetext{
1 Hereafter, following LL04, we will distinguish between inelastic and superelastic collisions. The former induce transitions towards higher energy levels ("exciting" collisions), the latter induce transitions towards lower energy levels ("de-exciting" collisions).
}

where $\xi$ and $\boldsymbol{\Omega}$ are the photon's frequency (in the atom rest frame) and propagation direction, respectively. The quantity $W_{K}\left(J_{\ell}, J_{u}\right)$ is the polarizability factor of the transition, given by (see Eq. (10.17) of LL04)

$W_{K}\left(J_{\ell}, J_{u}\right)=3\left(2 J_{u}+1\right)\left\{\begin{array}{ccc}1 & 1 & K \\ J_{u} & J_{u} & J_{\ell}\end{array}\right\}^{2}$,

with $J_{u}$ and $J_{\ell}$ the total angular momenta of the upper and lower level, respectively. The matrix $\left[P^{(K)}\left(\boldsymbol{\Omega}^{\prime}, \boldsymbol{\Omega}\right)\right]_{i j}(i, j=0,1,2,3)$ is the $K$ th multipole component of the scattering phase matrix. As shown in Eq. (10.21) of LL04, its explicit expression can be written in a very compact form in terms of the geometrical tensors $\mathcal{T}_{Q}^{K}(i, \boldsymbol{\Omega})$ introduced by Landi Degl'Innocenti (1984)

$\left[P^{(K)}\left(\mathbf{\Omega}^{\prime}, \mathbf{\Omega}\right)\right]_{i j}=\sum_{Q=-K}^{K}(-1)^{Q} \mathcal{T}_{Q}^{K}(i, \boldsymbol{\Omega}) \mathcal{T}_{-Q}^{K}\left(j, \boldsymbol{\Omega}^{\prime}\right)$.

The quantities $\Gamma_{R}, \Gamma_{I}$, and $\Gamma_{E}$ are the line broadening constants due to radiative decays, collisional de-excitation, and elastic collisions:

$\Gamma_{\mathrm{R}}=A_{u \ell}, \quad \Gamma_{\mathrm{I}}=C_{u \ell}, \quad \Gamma_{\mathrm{E}}=Q_{\mathrm{el}}$,

where $A_{u \ell}$ is the Einstein coefficient for spontaneous emission (i.e., the radiative de-excitation rate), $C_{u \ell}$ is the collisional deexcitation rate, and $Q_{\mathrm{el}}$ is the elastic collision rate. The quantity $D^{(K)}$ is the depolarizing rate due to elastic collisions, defined as in Eq. (7.102) of LL04 ${ }^{2}$. The profile $\phi$ is the line absorption profile, $v_{0}$ is the Bohr frequency corresponding to the transition between the upper and lower level, and $\delta\left(\xi-\xi^{\prime}\right)$ is the Dirac delta. The absorption profile $\phi$ is in general a Lorentzian

$\phi\left(v_{0}-\xi\right)=\frac{1}{\pi} \frac{\Gamma / 4 \pi}{\left(v_{0}-\xi\right)^{2}+(\Gamma / 4 \pi)^{2}}$,

characterized by the broadening constant

$\Gamma=\Gamma_{\mathrm{R}}+\Gamma_{\mathrm{I}}+\Gamma_{\mathrm{E}}$.

The redistribution matrix of Eq. (1) can also be written in the equivalent form

$$
\begin{aligned}
R_{i j}\left(\xi^{\prime}, \mathbf{\Omega}^{\prime} ; \xi, \mathbf{\Omega}\right)=\sum_{K=0}^{2}\{ & \alpha\left[R_{\mathrm{II}}^{(K)}\left(\xi^{\prime}, \mathbf{\Omega}^{\prime} ; \xi, \mathbf{\Omega}\right)\right]_{i j} \\
& \left.+\left(\beta^{(K)}-\alpha\right)\left[R_{\mathrm{III}}^{(K)}\left(\xi^{\prime}, \mathbf{\Omega}^{\prime} ; \xi, \mathbf{\Omega}\right)\right]_{i j}\right\}
\end{aligned}
$$

with

$$
\begin{aligned}
{\left[R_{\mathrm{II}}^{(K)}\left(\xi^{\prime}, \boldsymbol{\Omega}^{\prime} ; \xi, \boldsymbol{\Omega}\right)\right]_{i j}=} & (1-\epsilon) W_{K}\left(J_{\ell}, J_{u}\right)\left[P^{(K)}\left(\mathbf{\Omega}^{\prime}, \boldsymbol{\Omega}\right)\right]_{i j} \\
& \times \delta\left(\xi-\xi^{\prime}\right) \phi\left(v_{0}-\xi\right), \\
{\left[R_{\mathrm{III}}^{(K)}\left(\xi^{\prime}, \boldsymbol{\Omega}^{\prime} ; \xi, \boldsymbol{\Omega}\right)\right]_{i j}=} & (1-\epsilon) W_{K}\left(J_{\ell}, J_{u}\right)\left[P^{(K)}\left(\mathbf{\Omega}^{\prime}, \boldsymbol{\Omega}\right)\right]_{i j} \\
& \times \phi\left(v_{0}-\xi^{\prime}\right) \phi\left(v_{0}-\xi\right),
\end{aligned}
$$

and where the quantities $\alpha$ and $\beta^{(K)}$ are defined as

$$
\begin{aligned}
\alpha & =\frac{\Gamma_{\mathrm{R}}+\Gamma_{\mathrm{I}}}{\Gamma_{\mathrm{R}}+\Gamma_{\mathrm{I}}+\Gamma_{\mathrm{E}}}, \\
\beta^{(K)} & =\frac{\Gamma_{\mathrm{R}}+\Gamma_{\mathrm{I}}}{\Gamma_{\mathrm{R}}+\Gamma_{\mathrm{I}}+D^{(K)}} .
\end{aligned}
$$

2 The quantities $D^{(K)}$ and $\Gamma_{\mathrm{E}}$ are related to each other, but the exact relationship between them is still matter of debate. Approximate expressions relating $D^{(K)}$ and $\Gamma_{\mathrm{E}}$, obtained through simplified collisional models, can be found in Stenflo (1994) and in Chapter 5 of LL04. 
It should be observed that the factor $(1-\epsilon)$ appearing in Eqs. (8) and (9), with

$\epsilon=\frac{C_{u \ell}}{A_{u \ell}+C_{u \ell}}=\frac{\Gamma_{\mathrm{I}}}{\Gamma_{\mathrm{R}}+\Gamma_{\mathrm{I}}}$

the photon destruction probability, was implicitly contained in the factors

$\frac{\Gamma_{\mathrm{R}}}{\Gamma_{\mathrm{R}}+\Gamma_{\mathrm{I}}+\Gamma_{\mathrm{E}}}=(1-\epsilon) \alpha$

and

$\frac{\Gamma_{\mathrm{R}}}{\Gamma_{\mathrm{R}}+\Gamma_{\mathrm{I}}+D^{(K)}} \frac{\Gamma_{\mathrm{E}}-D^{(K)}}{\Gamma_{\mathrm{R}}+\Gamma_{\mathrm{I}}+\Gamma_{\mathrm{E}}}=(1-\epsilon)\left(\beta^{(K)}-\alpha\right)$

appearing in the righthand side of Eq. (1).

As shown by Eq. (7), in the atom rest frame the total redistribution matrix is composed of the linear combination of two terms, which describe coherent scattering processes $\left(R_{\mathrm{II}}\right)$, and scattering processes in the limit of complete frequency redistribution $\left(R_{\mathrm{III}}\right)$. The branching ratio for the coherent scattering contribution $(\alpha)$ can be interpreted as the probability that the photon (after being absorbed) is re-emitted before the atom suffers an elastic collision, provided that it is re-emitted at all, while the branching ratio for the CRD scattering contribution $\left(\beta^{(K)}-\alpha\right)$ represents the probability that the photon is re-emitted after the atom has suffered an elastic collision that, however, does not completely destroy atomic polarization (see Domke \& Hubeny 1988; Bommier 1997a,b, for more details). When polarization phenomena are considered, elastic collisions actually play a double role: on the one hand, they are responsible for frequency redistribution; on the other, they depolarize the atomic system. The sum of the branching ratios $\alpha$ and $\left(\beta^{(K)}-\alpha\right)$ is equal to 1 for the $K=0$ multipole component $\left(D^{(0)} \equiv 0\right)$ while, in the presence of depolarizing collisions, it is generally smaller than 1 for $K \neq 0$.

\section{Redistribution matrix for a two-term atom}

Quantum interference between different $J$-levels is known to produce significant observable effects on the scattering polarization signals in the wings of strong resonance lines such as $\mathrm{Ca}$ II $\mathrm{H} \& \mathrm{~K}, \mathrm{Na} \mathrm{I}_{1}$ and $\mathrm{D}_{2}, \mathrm{Mg}$ II $\mathrm{h} \& \mathrm{k}$, or $\mathrm{H}$ I Ly- $\alpha$ (e.g., Stenflo 1980; Stenflo \& Keller 1997; Landi Degl'Innocenti 1998; LL04; Belluzzi \& Trujillo Bueno 2011, 2012; Belluzzi et al. 2012). An atomic model accounting for the contribution of $J$-state interference, such as the multiterm model atom described in LL04, is therefore needed for the investigation of these lines. Unfortunately, the development of a self-consistent PRD theory for such an atomic model presents remarkable difficulties and has not been achieved yet.

In this work we consider the simpler case of a two-term model atom under the assumptions that the fine structure levels of the lower term are infinitely sharp, and that the magnetic sublevels of the lower term are evenly populated and no interference is present between them (unpolarized lower term). By analogy with the two-level atom case, we assume that in the atom rest frame the redistribution matrix for such a two-term model atom is given by a linear combination of two terms: one describing coherent scattering processes $\left(R_{\mathrm{II}}\right)$, and one describing scattering processes in the limit of complete frequency redistribution $\left(R_{\mathrm{III}}\right)$. The first goal of our work is to derive suitable expressions of $R_{\mathrm{II}}$ and $R_{\mathrm{III}}$ for the unmagnetized case. The limit of purely coherent scattering is investigated within the framework of the theoretical approach developed by Landi Degl'Innocenti et al. (1997), while the CRD limit is investigated within the framework of the quantum theory of polarization presented in LL04.

\section{1. $R_{\text {III }}$ redistribution matrix}

The limit of CRD is reached when collisions are very efficient in redistributing the photon frequency during the scattering process. A rigorous treatment of collisional processes is therefore essential for a correct derivation of the $R_{\text {III }}$ redistribution matrix. In a two-level atom, frequency redistribution (in the atom rest frame) is due to long-range collisions, typically with neutral hydrogen atoms, inducing transitions among the various magnetic sublevels of a given $J$-level (elastic collisions). In a twoterm atom, on the other hand, frequency redistribution is also produced by collisions (due to either electrons, protons, or neutral hydrogen) inducing transitions between different $J$-levels of the same term. Taking properly into account the effect of these collisions (hereafter referred to as weakly inelastic collisions) presents, however, an intrinsic difficulty when working within the framework of the redistribution matrix formalism. This is because the derivation of the redistribution matrix requires an analytical solution of the statistical equilibrium equations, which in general cannot be achieved if such collisions are taken into account. In this work, we derive an approximate expression of the $R_{\mathrm{III}}$ redistribution matrix, under the assumption of CRD in the observer's frame. In this section, we point out the approximations it is based on, and we discuss its physical meaning, clarifying under which assumptions the effect of collisions is taken into account.

We deduce the $R_{\mathrm{III}}$ redistribution matrix starting from the expression of the emission coefficient of a two-term atom (with unpolarized lower term) given by Eq. (68) of Belluzzi et al. (2013). This emission coefficient has been obtained within the framework of the theory of polarization presented in LL04, and accounts for the effect of inelastic and superelastic collisions with electrons, inducing transitions between $J$-levels pertaining to different terms. For convenience, the explicit expression of this emission coefficient, which has been obtained in the absence of magnetic fields and neglecting stimulated emission, is rewritten below:

$$
\begin{aligned}
& \varepsilon_{i}(\xi, \boldsymbol{\Omega})=k_{L} \frac{2 L_{u}+1}{2 S+1} \sum_{K Q} \sum_{J_{u} J_{u}^{\prime} J_{\ell}}(-1)^{S-L_{\ell}+J_{u}+J_{u}^{\prime}+J_{\ell}+K+Q} \\
& \times 3\left(2 J_{u}+1\right)\left(2 J_{u}^{\prime}+1\right)\left(2 J_{\ell}+1\right) \\
& \times\left\{\begin{array}{ccc}
L_{u} & L_{\ell} & 1 \\
J_{\ell} & J_{u} & S
\end{array}\right\}\left\{\begin{array}{ccc}
L_{u} & L_{\ell} & 1 \\
J_{\ell} & J_{u}^{\prime} & S
\end{array}\right\}\left\{\begin{array}{ccc}
1 & 1 & K \\
J_{u} & J_{u}^{\prime} & J_{\ell}
\end{array}\right\} \\
& \times\left\{\begin{array}{ccc}
1 & 1 & K \\
L_{u} & L_{u} & L_{\ell}
\end{array}\right\}\left\{\begin{array}{ccc}
L_{u} & L_{u} & K \\
J_{u} & J_{u}^{\prime} & S
\end{array}\right\} \mathcal{T}_{Q}^{K}(i, \mathbf{\Omega}) \\
& \times \frac{1}{2} \frac{\Phi\left(v_{J_{u} J_{\ell}}-\xi\right)+\Phi\left(v_{J_{u}^{\prime} J_{\ell}}-\xi\right)^{*}}{1+\epsilon^{\prime}+2 \pi \mathrm{i} v_{J_{u}^{\prime} J_{u}} / A\left(L_{u} \rightarrow L_{\ell}\right)} J_{-Q}^{K}\left(\xi_{0}^{\prime}\right) \\
& +\frac{\epsilon^{\prime}}{1+\epsilon^{\prime}} k_{L} B_{T}\left(v_{0}\right) \varphi(\xi) \delta_{i 0},
\end{aligned}
$$

with $i=0,1,2$, and 3 , standing for Stokes $I, Q, U$, and $V$, respectively, and where $\xi$ and $\boldsymbol{\Omega}$ are the frequency (in the atom rest frame) and propagation direction, respectively, of the emitted radiation. The quantity $k_{L}$ is the frequency-integrated absorption coefficient of the multiplet, given by

$k_{L}=\frac{h v_{0}}{4 \pi} \mathbb{N}_{\ell} B\left(L_{\ell} \rightarrow L_{u}\right)$,

where $v_{0}$ is the Bohr frequency corresponding to the energy separation between the centers of gravity of the two terms, $\mathbb{N}_{\ell}$ is the number density of atoms in the lower term, and $B\left(L_{\ell} \rightarrow L_{u}\right)$ is the Einstein coefficient for absorption from the lower to the 
upper term. The quantum numbers $L$ and $S$ are the orbital angular momentum and spin, respectively, characterizing the upper and lower terms (we always assume that the atom is described within the $L-S$ coupling scheme). The complex emission profile $\Phi\left(v_{J_{u} J_{\ell}}-\xi\right)$ is defined by

$\Phi\left(v_{J_{u} J_{\ell}}-\xi\right)=\phi\left(v_{J_{u} J_{\ell}}-\xi\right)+\mathrm{i} \psi\left(v_{J_{u} J_{\ell}}-\xi\right)$,

with

$\phi\left(v_{J_{u} J_{\ell}}-\xi\right)=\frac{1}{\pi} \frac{\Gamma / 4 \pi}{\left(v_{J_{u} J_{\ell}}-\xi\right)^{2}+(\Gamma / 4 \pi)^{2}}$,

the Lorentzian profile, and

$\psi\left(v_{J_{u} J_{\ell}}-\xi\right)=\frac{1}{\pi} \frac{\left(v_{J_{u} J_{\ell}}-\xi\right)}{\left(v_{J_{u} J_{\ell}}-\xi\right)^{2}+(\Gamma / 4 \pi)^{2}}$,

the associated dispersion profile. These profiles are centered at the frequencies $v_{J_{u} J_{\ell}}$ of the various fine structure components of the multiplet, and are characterized by the broadening constant

$\Gamma=\Gamma_{\mathrm{R}}+\Gamma_{\mathrm{I}}+\Gamma_{\mathrm{E}}$.

In a two-term atom, the line broadening constants due to radiative decays $\left(\Gamma_{R}\right)$, collisional decays $\left(\Gamma_{I}\right)$, and elastic collisions $\left(\Gamma_{\mathrm{E}}\right)$ are given by

$\Gamma_{\mathrm{R}}=A\left(L_{u} \rightarrow L_{\ell}\right), \quad \Gamma_{\mathrm{I}}=C_{S}\left(L_{u} \rightarrow L_{\ell}\right), \quad \Gamma_{\mathrm{E}}=Q_{\mathrm{el}}$,

where $A\left(L_{u} \rightarrow L_{\ell}\right)=\sum_{J_{\ell}} A\left(J_{u} \rightarrow J_{\ell}\right)$ is the Einstein coefficient for spontaneous emission from the upper to the lower term (see footnote 2 at page 314 of LL04), $C_{S}\left(L_{u} \rightarrow L_{\ell}\right)=\sum_{J_{\ell}} C_{S}\left(J_{u} \rightarrow\right.$ $\left.J_{\ell}\right)$ is the superelastic collision rate for the transition from the upper to the lower term (see Eq. (38) of Belluzzi et al. 2013), and where $Q_{\mathrm{el}}$ is the rate of elastic collisions (we assume that the line broadening due to elastic collisions is the same for all the lines of the multiplet). The quantity $\epsilon^{\prime}$ is defined as

$\epsilon^{\prime}=\frac{C_{S}\left(L_{u} \rightarrow L_{\ell}\right)}{A\left(L_{u} \rightarrow L_{\ell}\right)}=\frac{\Gamma_{\mathrm{I}}}{\Gamma_{\mathrm{R}}}$.

We observe that this quantity is connected to $\epsilon=\Gamma_{I} /\left(\Gamma_{R}+\Gamma_{I}\right)$ (see Eq. (12)) by the relation

$\epsilon=\frac{\epsilon^{\prime}}{1+\epsilon^{\prime}}$.

The frequency $v_{J_{u}^{\prime} J_{u}}$ is the Bohr frequency corresponding to the energy separation between the levels $J_{u}^{\prime}$ and $J_{u}$. The tensor $J_{Q}^{K}\left(\xi_{0}^{\prime}\right)$, which describes the incident radiation field, is given by (see Eq. (5.157) of LL04)

$J_{Q}^{K}\left(\xi_{0}^{\prime}\right)=\oint \frac{\mathrm{d} \mathbf{\Omega}^{\prime}}{4 \pi} \sum_{j=0}^{3} \mathcal{T}_{Q}^{K}\left(j, \boldsymbol{\Omega}^{\prime}\right) I_{j}\left(\xi_{0}^{\prime}, \mathbf{\Omega}^{\prime}\right)$,

with $I_{j}\left(\xi_{0}^{\prime}, \Omega^{\prime}\right)$ the Stokes parameters of the incident radiation field at an arbitrary frequency $\xi_{0}^{\prime}$ within the multiplet. We recall that the theory of LLO4 is strictly valid under the so-called flat-spectrum approximation ${ }^{3}$. In a two-term atom, where quantum interference between different $J$-levels is taken into account,

\footnotetext{
3 As previously pointed out, the theory of LL04 is based on a lowest order expansion within the framework of quantum electrodynamics. As discussed in Sect. 6.5 of LL04, the equations that are obtained within this theoretical framework are strictly correct only if the incident radiation field is flat (i.e., independent of frequency) across a spectral interval larger than the frequency separation between levels between which quantum interference is taken into account, and larger than the inverse lifetime of each level (flat-spectrum approximation). If this requirement is not met, inconsistencies in the radiation emitted in scattering events may in general be found (see also footnote 1 of Landi Degl'Innocenti et al. 1997).
}

this approximation requires the incident field to be flat across the whole multiplet. For this reason, in Eq. (13) it is sufficient to express the radiation field tensor $J_{Q}^{K}$ at a single, arbitrary frequency $\xi_{0}^{\prime}$ within the multiplet.

The last term in the righthand side of Eq. (13) represents the contribution to the emission coefficient coming from atoms that are collisionally excited. Since collisions are assumed to be isotropic, this term only contributes to Stokes $I$. The profile

$\varphi(\xi)=\sum_{J_{u} J_{\ell}} \frac{\left(2 J_{\ell}+1\right)\left(2 J_{u}+1\right)}{2 S+1}\left\{\begin{array}{ccc}L_{u} & L_{\ell} & 1 \\ J_{\ell} & J_{u} & S\end{array}\right\}^{2} \phi\left(v_{J_{u} J_{\ell}}-\xi\right)$

is the normalized absorption profile of the multiplet (in the absence of magnetic fields, and under the hypothesis of unpolarized lower term). The quantity $B_{T}\left(v_{0}\right)$ is the Planck function in the Wien limit (consistently with the hypothesis of neglecting stimulated emission), at the temperature $T$.

As previously pointed out, the expression of the emission coefficient of Eq. (13) is strictly valid under the assumption that the incident radiation field is flat across the whole multiplet (flatspectrum approximation). This is a very restrictive condition, which is generally not verified in a stellar atmosphere. Moreover, the detailed spectral structure of the incident field is the key physical aspect in the investigation of PRD phenomena. For this reason, we introduce the following important approximation: we take the frequency dependence of the incident field fully into account in the problem, but we evaluate Eq. (13) in terms of the frequency-integrated radiation field tensor, $\bar{J}_{Q}^{K}$, obtained by averaging the monochromatic radiation field tensor, $J_{Q}^{K}\left(\xi^{\prime}\right)$, over the absorption profile of the multiplet defined in Eq. (23):

$\bar{J}_{Q}^{K}=\int \mathrm{d} \xi^{\prime} \varphi\left(\xi^{\prime}\right) J_{Q}^{K}\left(\xi^{\prime}\right)$.

Substituting the explicit expression of $\bar{J}_{Q}^{K}$ into Eq. (13), the emission coefficient can be written as:

$$
\begin{aligned}
\varepsilon_{i}(\xi, \boldsymbol{\Omega})= & k_{L} \int \mathrm{d} \xi^{\prime} \oint \frac{\mathrm{d} \mathbf{\Omega}^{\prime}}{4 \pi} \sum_{j=0}^{3}\left[R_{\mathrm{III}}\left(\xi^{\prime}, \boldsymbol{\Omega}^{\prime} ; \xi, \boldsymbol{\Omega}\right)\right]_{i j} I_{j}\left(\xi^{\prime}, \mathbf{\Omega}^{\prime}\right) \\
& +\frac{\epsilon^{\prime}}{1+\epsilon^{\prime}} k_{L} B_{T}\left(v_{0}\right) \varphi(\xi) \delta_{i 0}
\end{aligned}
$$

with

$$
\begin{aligned}
{\left[R_{\mathrm{III}}(\right.} & \left.\left.\xi^{\prime}, \boldsymbol{\Omega}^{\prime} ; \xi, \boldsymbol{\Omega}\right)\right]_{i j}=\frac{2 L_{u}+1}{2 S+1} \sum_{K} \sum_{J_{u} J_{u}^{\prime} J_{\ell}}(-1)^{S-L_{\ell}+J_{u}+J_{u}^{\prime}+J_{\ell}+K} \\
& \times 3\left(2 J_{u}+1\right)\left(2 J_{u}^{\prime}+1\right)\left(2 J_{\ell}+1\right) \\
& \times\left\{\begin{array}{lll}
L_{u} & L_{\ell} & 1 \\
J_{\ell} & J_{u} & S
\end{array}\right\}\left\{\begin{array}{lll}
L_{u} & L_{\ell} & 1 \\
J_{\ell} & J_{u}^{\prime} & S
\end{array}\right\}\left\{\begin{array}{ccc}
1 & 1 & K \\
J_{u} & J_{u}^{\prime} & J_{\ell}
\end{array}\right\} \\
& \times\left\{\begin{array}{ccc}
1 & 1 & K \\
L_{u} & L_{u} & L_{\ell}
\end{array}\right\}\left\{\begin{array}{ccc}
L_{u} & L_{u} & K \\
J_{u} & J_{u}^{\prime} & S
\end{array}\right\}\left[P^{(K)}\left(\boldsymbol{\Omega}^{\prime}, \boldsymbol{\Omega}\right)\right]_{i j} \\
& \times \frac{1}{2} \frac{\Phi\left(v_{J_{u} J_{\ell}}-\xi\right)+\Phi\left(v_{J_{u}^{\prime} J_{\ell}}-\xi\right)^{*}}{1+\epsilon^{\prime}+2 \pi \mathrm{i} v_{J_{u}^{\prime} J_{u}} / A\left(L_{u} \rightarrow L_{\ell}\right)} \varphi\left(\xi^{\prime}\right)
\end{aligned}
$$

Equation (26) represents an approximate expression of the $R_{\mathrm{III}}$ redistribution matrix. Unfortunately, a general CRD theory for a two-term atom, obtained by taking the effect of elastic and weakly inelastic collisions into account, for an arbitrary incident radiation field, is not available yet. The inclusion of collisions in the theoretical framework of Landi Degl'Innocenti et al. (1997) 
is still under investigation, while the application of the CRD theory of Landi Degl'Innocenti (1983) to the case of a two-term atom illuminated by an arbitrary (non spectrally flat) radiation field may in general lead to inconsistencies (see footnote 3 ). For these reasons, we believe that the CRD theory of LL04, despite of the limitation due to the flat-spectrum approximation, is at the moment the most robust framework for deriving an approximate expression of $R_{\mathrm{III}}$. The introduction of the frequency-integrated radiation field tensor $\bar{J}_{Q}^{K}$ in Eq. (13), which allows us to apply the statistical equilibrium equations of LL04, still taking the frequency dependence of the radiation field into account in the radiative transfer equations, is certainly questionable. Indeed, this is just an ansatz, whose main support is the fact that the ensuing expression of $R_{\mathrm{III}}$ has a well defined physical meaning, and whose justification is better substantiated by the assumption of $\mathrm{CRD}$ in the observer's frame that we will make in the following.

The redistribution matrix of Eq. (26) describes scattering events in the limit in which collisions are able to redistribute the photon frequency not only within a single spectral line, but across the whole multiplet. This extreme CRD limit is reached when collisions are extremely efficient in inducing transitions between magnetic sublevels pertaining either to the same $J$-level or to different $J$-levels of the upper term. This is a strong approximation, especially when the energy separation among the various fine structure $J$-levels is large. Indeed, it is strictly justified only when the number density of colliders is very high (i.e., when $\Gamma_{\mathrm{E}} \gg \Gamma_{\mathrm{R}}+\Gamma_{\mathrm{I}}$ ), and when the energy of the colliding particles, on the order of $k_{\mathrm{B}} T$, with $k_{\mathrm{B}}$ the Boltzmann constant and $T$ the temperature of the plasma, is much higher than the fine structure splitting of the terms ${ }^{4}$.

The $R_{\text {III }}$ redistribution matrix of Eq. (26) describes the following physical process. We have a two-term atom which is initially in the lower level $J_{\ell}$. It is then excited in a state $\left(J_{u}-J_{u}^{\prime}\right)$ (a coherence, in general), and it finally de-excites towards a lower level $J_{\ell}^{\prime}$ (Raman scattering). While the atom is in the excited state, elastic and weakly inelastic collisions induce transitions between different magnetic sublevels pertaining either to the same $J$-level or to different $J$-levels of the upper term. In particular, they can induce transitions between the coherence $\left(J_{u}-J_{u}^{\prime}\right)$ and the coherence $\left(J_{u}-J_{u}^{\prime \prime}\right)$ or $\left(J_{u}^{\prime \prime}-J_{u}^{\prime}\right)$. Equation (26) explicitly contains a sum over the quantum numbers $\left(J_{\ell}, J_{u}, J_{u}^{\prime}\right)$, while a sum over $\left(J_{\ell}, J_{u}\right)$ is implicitly contained in the absorption profile $\varphi\left(\xi^{\prime}\right)$ (see Eq. (23)). If the two sums are not factorized, the $R_{\mathrm{III}}$ redistribution matrix would explicitly show a sum over the quantum numbers $\left(J_{\ell}, J_{\ell}^{\prime}, J_{u}, J_{u}^{\prime}\right.$, and $\left.J_{u}^{\prime \prime}\right)$, which is actually required for describing the above-mentioned scattering process.

Besides redistributing the photon frequency during the scattering process, elastic and weakly inelastic collisions also contribute to destroy atomic polarization. Expressions of the depolarizing rates due to elastic and weakly inelastic collisions with neutral hydrogen atoms in multilevel systems can be found in Sahal-Bréchot et al. (2007). Although the CRD limit described by our $R_{\mathrm{III}}$ redistribution matrix is strictly valid when collisional processes are extremely efficient, in this work the depolarizing effect of collisions is neglected. This is not fully consistent, but the inclusion of this effect in a two-term atom is not straightforward, and its analysis goes beyond the scope of this investigation. As previously discussed, a first difficulty is due to the fact

\footnotetext{
4 Under such circumstances, collisions between different $J$-levels of the same term (referred in this paper to as "weakly inelastic" collisions) can be rightly considered as "elastic" collisions (i.e., collisions between levels whose energy separation is much smaller than the energy of the colliding particle).
}

that the effect of weakly inelastic collisions cannot be rigorously accounted for within the framework of the redistribution matrix formalism. A second difficulty concerns $J$-state interference. In a two-level atom, elastic collisions contribute to equalize the population of the various magnetic sublevels of a given $J$-level, and to relax quantum interference between pairs of them. This depolarizing effect is described through the rate $D^{(K)}$ discussed in Sect. 2. In a two-term atom, on the other hand, also $J$-state interference is present, and it is still unclear what is the impact of elastic and weakly inelastic collisions on such interference.

In the limit of a two-level atom (which can be obtained by setting $S=0, L_{u}=J_{u}$ and $L_{\ell}=J_{\ell}$ ), Eq. (26) reduces to

$$
\begin{gathered}
{\left[R_{\mathrm{III}}\left(\xi^{\prime}, \boldsymbol{\Omega}^{\prime} ; \xi, \boldsymbol{\Omega}\right)\right]_{i j}^{\mathrm{two}-\mathrm{lev}}=\sum_{K} 3\left(2 J_{u}+1\right)\left\{\begin{array}{ccc}
1 & 1 & K \\
J_{u} & J_{u} & J_{\ell}
\end{array}\right\}^{2}} \\
\times\left[P^{(K)}\left(\boldsymbol{\Omega}^{\prime}, \boldsymbol{\Omega}\right)\right]_{i j} \frac{1}{1+\epsilon^{\prime}} \phi\left(v_{J_{u} J_{\ell}}-\xi\right) \phi\left(v_{J_{u} J_{\ell}}-\xi^{\prime}\right) .
\end{gathered}
$$

Recalling the definition of the polarizability factor (see Eq. (2)), and the relation between the quantities $\epsilon$ and $\epsilon^{\prime}$ (see Eq. (21)), it is easy to verify that the various multipole terms in the righthand side of Eq. (27) coincide with Eq. (9). We thus recover the redistribution matrix of Eq. (1) derived by Bommier (1997a), in the limit of CRD scattering, and in the absence of depolarizing collisions (i.e., in the limit of $\alpha=0$ and $\left.\beta^{(K)}=1\right)^{5}$.

The redistribution matrix given by Eq. (26) is valid in the atom rest frame. In order to find the corresponding expression in the observer's frame, one has to take the Doppler effect into account for the given velocity distribution of the atoms. However, taking the previously introduced approximations into account, we make the assumption of CRD also in the observer's frame. Under this assumption, the expression of $R_{\mathrm{III}}$ in the observer's frame is still given by Eq. (26), with the only difference that the functions $\phi$ and $\psi$ appearing in the complex emission profile $\Phi$ (see Eq. (15)) and in the absorption profile $\varphi$ (see Eq. (23)) are now the Voigt and the Faraday-Voigt profiles, respectively. The assumption of CRD in the observer's frame has been proved to be very good as far as the emergent intensity is concerned (e.g., Mihalas 1978). For the case of a two-level atom, an analysis of the differences coming out from the application of the exact and approximate observer's frame expressions of $R_{\mathrm{III}}$ in polarization studies has been carried out by Bommier (1997b).

As previously mentioned, in our applications we consider a total redistribution matrix given by a linear combination of $R_{\mathrm{II}}$ and $R_{\mathrm{III}}$ (see Sect. 3.3). It is worth to observe that the core of strong resonance lines forms high in the atmosphere, where the number density of perturbers is relatively low, and scattering is practically coherent in the atom rest frame. The impact of $R_{\mathrm{III}}$ is thus expected to be very small (or negligible) in such spectral regions, becoming more important in the wings of the lines. Applying an approximate expression of $R_{\mathrm{III}}$ is not expected therefore to compromise the correct modeling of scattering polarization in the core of strong resonance lines. On the other hand, it can provide qualitative information concerning the effects of collisional redistribution on the line wing polarization.

5 This limit is reached for $\Gamma_{\mathrm{E}} \gg \Gamma_{\mathrm{R}}+\Gamma_{\mathrm{I}}$, and for $D^{(K)}=0$; that is, when the frequency of elastic collisions is much higher than the inverse lifetime of the excited level (i.e., when the atom, once excited, undergoes a large number of elastic collisions before re-emitting the photon), and when the elastic collisions do not destroy the atomic polarization. 


\section{2. $R_{\|}$redistribution matrix}

A suitable theoretical approach for describing scattering polarization in the limit of purely coherent scattering in the atom rest frame is the one developed by Landi Degl'Innocenti et al. (1997). In this work, the authors neglect any kind of collisions, and derive the redistribution matrix, in the atom rest frame, for different atomic models. In particular, they derive the following redistribution matrix for a two-term atom with unpolarized lower term and infinitely sharp lower levels

$$
\begin{aligned}
{\left[R_{\mathrm{II}}\right.} & \left.\left.\xi^{\prime}, \boldsymbol{\Omega}^{\prime} ; \xi, \boldsymbol{\Omega}\right)\right]_{i j}=\frac{2 L_{u}+1}{2 S+1} \sum_{K} \sum_{J_{u} J_{u}^{\prime}} \sum_{J_{\ell} J_{\ell}^{\prime}}(-1)^{J_{\ell}-J_{\ell}^{\prime}} \\
& \times 3\left(2 J_{u}+1\right)\left(2 J_{u}^{\prime}+1\right)\left(2 J_{\ell}+1\right)\left(2 J_{\ell}^{\prime}+1\right) \\
& \times\left\{\begin{array}{ccc}
L_{u} & L_{\ell} & 1 \\
J_{\ell} & J_{u} & S
\end{array}\right\}\left\{\begin{array}{ccc}
L_{u} & L_{\ell} & 1 \\
J_{\ell} & J_{u}^{\prime} & S
\end{array}\right\}\left\{\begin{array}{ccc}
L_{u} & L_{\ell} & 1 \\
J_{\ell}^{\prime} & J_{u} & S
\end{array}\right\}\left\{\begin{array}{ccc}
L_{u} & L_{\ell} & 1 \\
J_{\ell}^{\prime} & J_{u}^{\prime} & S
\end{array}\right\} \\
& \times\left\{\begin{array}{ccc}
K & J_{u}^{\prime} & J_{u} \\
J_{\ell} & 1 & 1
\end{array}\right\}\left\{\begin{array}{ccc}
K & J_{u}^{\prime} & J_{u} \\
J_{\ell}^{\prime} & 1 & 1
\end{array}\right\}\left[P^{(K)}\left(\boldsymbol{\Omega}^{\prime}, \boldsymbol{\Omega}\right)\right]_{i j} \\
& \left.\times \frac{1}{\pi} \frac{A\left(L_{u} \rightarrow L_{\ell}\right)}{4 \pi}+\mathrm{i}\left(v_{J_{u}^{\prime} J_{\ell}}-\xi\right)\right]\left[\frac{A\left(L_{u} \rightarrow L_{\ell}\right)}{4 \pi}-\mathrm{i}\left(v_{J_{u} J_{\ell}}-\xi\right)\right] \\
& \times \delta\left(\xi-\xi^{\prime}-v_{\left.J_{\ell}^{\prime} J_{\ell}\right),}\right.
\end{aligned}
$$

where the matrix $\left[P^{(K)}\left(\boldsymbol{\Omega}^{\prime}, \boldsymbol{\Omega}\right)\right]_{i j}$ is defined in Eq. (3). The Dirac delta assures the coherency of scattering (required by energy conservation, in the absence of collisions, in the atom rest frame), the term $v_{J_{\ell}^{\prime} J_{\ell}}$ takes Raman scattering into account. Recalling the definition of the complex profile $\Phi\left(v_{J_{u} J_{\ell}}-\xi\right)$ (see Eq. (15)), the profile appearing in the last two lines in the righthand side of Eq. (28) can be rewritten as

$$
\frac{1}{2} \frac{\Phi\left(v_{J_{u} J_{\ell}}-\xi\right)+\Phi\left(v_{J_{u}^{\prime} J_{\ell}}-\xi\right)^{*}}{1+2 \pi \mathrm{i} v_{J_{u}^{\prime} J_{u}} / A\left(L_{u} \rightarrow L_{\ell}\right)} \delta\left(\xi-\xi^{\prime}-v_{J_{\ell}^{\prime} J_{\ell}}\right)
$$

where the Lorentzian profile $\phi$ and the associated dispersion profile $\psi$ appearing in the complex profile $\Phi$ are characterized by a broadening constant $\Gamma=\Gamma_{\mathrm{R}}=A\left(L_{u} \rightarrow L_{\ell}\right)$ (only radiative broadening is considered since collisions are neglected). It is of interest to mention that the same expression for $R_{\mathrm{II}}$ can be obtained from the Kramers-Heisenberg scattering formula (Smitha et al. 2011).

As previously pointed out, the $R_{\mathrm{II}}$ redistribution matrix of Eq. (28) has been derived in the atom rest frame, neglecting any kind of collisions. A self-consistent generalization of the theoretical approach developed by Landi Degl'Innocenti et al. (1997) in order to account for the effect of collisions is still under investigation. On the other hand, the effect of inelastic and superelastic collisions with electrons, inducing transitions between $J$-levels of different terms, has to be included in $R_{\mathrm{II}}$ in order to take into account that only a fraction of the atoms is radiatively excited and is thus described through a redistribution matrix (collisionally excited atoms contribute to the emission coefficient through the thermal term shown in the righthand side of Eq. (13)). We account for the effect of such collisions through the quantity $\epsilon^{\prime}$ (see Eq. (20)) that we introduce in the expression of $R_{\mathrm{II}}$ in analogy with the case of $R_{\mathrm{III}}$. Recalling Eq. (26) we have:

$$
\begin{aligned}
{\left[R_{\mathrm{II}}\right.} & \left.\left(\xi^{\prime}, \boldsymbol{\Omega}^{\prime} ; \xi, \boldsymbol{\Omega}\right)\right]_{i j}=\frac{2 L_{u}+1}{2 S+1} \sum_{K} \sum_{J_{u} J_{u}^{\prime}} \sum_{J_{\ell} J_{\ell}^{\prime}}(-1)^{J_{\ell}-J_{\ell}^{\prime}} \\
& \times 3\left(2 J_{u}+1\right)\left(2 J_{u}^{\prime}+1\right)\left(2 J_{\ell}+1\right)\left(2 J_{\ell}^{\prime}+1\right) \\
& \times\left\{\begin{array}{ccc}
L_{u} & L_{\ell} & 1 \\
J_{\ell} & J_{u} & S
\end{array}\right\}\left\{\begin{array}{ccc}
L_{u} & L_{\ell} & 1 \\
J_{\ell} & J_{u}^{\prime} & S
\end{array}\right\}\left\{\begin{array}{ccc}
L_{u} & L_{\ell} & 1 \\
J_{\ell}^{\prime} & J_{u} & S
\end{array}\right\} \\
& \times\left\{\begin{array}{ccc}
L_{u} & L_{\ell} & 1 \\
J_{\ell}^{\prime} & J_{u}^{\prime} & S
\end{array}\right\}\left\{\begin{array}{ccc}
K & J_{u}^{\prime} & J_{u} \\
J_{\ell} & 1 & 1
\end{array}\right\}\left\{\begin{array}{ccc}
K & J_{u}^{\prime} & J_{u} \\
J_{\ell}^{\prime} & 1 & 1
\end{array}\right\} \\
& \times\left[P^{(K)}\left(\boldsymbol{\Omega}^{\prime}, \boldsymbol{\Omega}\right)\right]_{i j} \frac{1}{2} \frac{\Phi\left(v_{J_{u} J_{\ell}}-\xi\right)+\Phi\left(v_{\left.J_{u}^{\prime} J_{\ell}-\xi\right)^{*}}\right.}{1+\epsilon^{\prime}+2 \pi \mathrm{i} v_{J_{u}^{\prime} J_{u}} / A\left(L_{u} \rightarrow L_{\ell}\right)} \\
& \times \delta\left(\xi-\xi^{\prime}-v_{J_{\ell}^{\prime} J_{\ell}}\right) .
\end{aligned}
$$

In the limit of a two-level atom (which can be obtained by setting $S=0, L_{u}=J_{u}$ and $L_{\ell}=J_{\ell}$ ), Eq. (30) reduces to

$$
\begin{gathered}
{\left[R_{\mathrm{II}}\left(\xi^{\prime}, \boldsymbol{\Omega}^{\prime} ; \xi, \boldsymbol{\Omega}\right)\right]_{i j}^{\mathrm{two}-\mathrm{lev}}=\sum_{K} 3\left(2 J_{u}+1\right)\left\{\begin{array}{ccc}
1 & 1 & K \\
J_{u} & J_{u} & J_{\ell}
\end{array}\right\}^{2}} \\
\times\left[P^{(K)}\left(\boldsymbol{\Omega}^{\prime}, \boldsymbol{\Omega}\right)\right]_{i j} \frac{1}{1+\epsilon^{\prime}} \phi\left(v_{J_{u} J_{\ell}}-\xi\right) \delta\left(\xi-\xi^{\prime}\right) .
\end{gathered}
$$

It can be easily verified that the various multipole terms in the righthand side of Eq. (31) coincide with Eq. (8). We thus recover the redistribution matrix of Eq. (1), in the limit of purely coherent scattering (i.e., in the limit of $\alpha=1)^{6}$.

Equation (30) is valid in the atom rest frame. The expression of $R_{\mathrm{II}}$ in the observer's frame, obtained by taking Doppler redistribution into account, can be calculated following a derivation analogous to the one presented in Hummer (1962). A detailed derivation can be found in Appendix A. Indicating with $v^{\prime}$ and $v$ the frequencies of the incoming and outgoing photons in the observer's frame, and assuming that the atoms have a Maxwellian velocity distribution, we obtain

$$
\begin{aligned}
{\left[R_{\mathrm{II}}\right.} & \left.\left(v^{\prime}, \boldsymbol{\Omega}^{\prime} ; v, \boldsymbol{\Omega}\right)\right]_{i j}=\frac{2 L_{u}+1}{2 S+1} \sum_{K} \sum_{J_{u} J_{u}^{\prime}} \sum_{J_{\ell} J_{\ell}^{\prime}}(-1)^{J_{\ell}-J_{\ell}^{\prime}} \\
& \times 3\left(2 J_{u}+1\right)\left(2 J_{u}^{\prime}+1\right)\left(2 J_{\ell}+1\right)\left(2 J_{\ell}^{\prime}+1\right) \\
& \times\left\{\begin{array}{ccc}
L_{u} & L_{\ell} & 1 \\
J_{\ell} & J_{u} & S
\end{array}\right\}\left\{\begin{array}{ccc}
L_{u} & L_{\ell} & 1 \\
J_{\ell} & J_{u}^{\prime} & S
\end{array}\right\}\left\{\begin{array}{ccc}
L_{u} & L_{\ell} & 1 \\
J_{\ell}^{\prime} & J_{u} & S
\end{array}\right\} \\
& \times\left\{\begin{array}{ccc}
L_{u} & L_{\ell} & 1 \\
J_{\ell}^{\prime} & J_{u}^{\prime} & S
\end{array}\right\}\left\{\begin{array}{ccc}
K & J_{u}^{\prime} & J_{u} \\
J_{\ell} & 1 & 1
\end{array}\right\}\left\{\begin{array}{ccc}
K & J_{u}^{\prime} & J_{u} \\
J_{\ell}^{\prime} & 1 & 1
\end{array}\right\} \\
& \times\left[P^{(K)}\left(\boldsymbol{\Omega}^{\prime}, \boldsymbol{\Omega}\right)\right]_{i j} \frac{1}{\pi \Delta v_{\mathrm{D}}^{2} \sin \Theta} \exp \left[-\frac{\left(v^{\prime}-v+v_{\left.J_{\ell}^{\prime} J_{\ell}\right)^{2}}\right.}{4 \Delta v_{\mathrm{D}}^{2} \sin ^{2}(\Theta / 2)}\right] \\
& \times \frac{1}{1+\epsilon^{\prime}+2 \pi \mathrm{i} v_{J_{u}^{\prime} J_{u}} / A\left(L_{u} \rightarrow L_{\ell}\right)} \\
& \times \frac{1}{2}\left[W\left(\frac{a}{\cos (\Theta / 2)}, \frac{v_{J_{u} J_{\ell}}+v_{J_{u} J_{\ell}^{\prime}}^{\prime}}{2 \cos (\Theta / 2)}\right)^{\frac{a}{\cos (\Theta)}}\right. \\
& \left.+W\left(\frac{v_{J_{u}^{\prime} J_{\ell}}+v_{J_{u}^{\prime} J_{\ell}^{\prime}}^{\prime}}{2 \cos (\Theta / 2)}\right)^{*}\right],
\end{aligned}
$$

where $\Theta$ is the scattering angle and $\Delta \nu_{\mathrm{D}}$ is the Doppler width (we assume that it is the same for all the lines of the multiplet). The function $W$ is defined as

$W(a, v)=H(a, v)+\mathrm{i} L(a, v)$,

6 This limit is reached for $\Gamma_{\mathrm{E}}=0$ (i.e., in the absence of elastic collisions). 
where $H$ is the Voigt function, $L$ the associated dispersion profile, and $a=\Gamma / 4 \pi \Delta v_{\mathrm{D}}$ the damping parameter. The reduced frequencies are given by

$v_{J_{a} J_{b}}=\frac{\left(v_{J_{a} J_{b}}-v\right)}{\Delta v_{\mathrm{D}}}, \quad v_{J_{a} J_{b}}^{\prime}=\frac{\left(v_{J_{a} J_{b}}-v^{\prime}\right)}{\Delta v_{\mathrm{D}}}$.

The numerical calculation of this redistribution matrix is rather demanding since the angular and frequency dependencies cannot be factorized as in the atom rest frame. For this reason, it is customary to work with an approximate expression, obtained by averaging the frequency redistribution part of the redistribution matrix over all the possible propagation directions $\boldsymbol{\Omega}^{\prime}$ and $\boldsymbol{\Omega}$ of the incoming and outgoing photons (see, Rees \& Saliba 1982). Since the frequency redistribution part of the redistribution matrix only depends on the scattering angle $\Theta$, such average can be easily reduced to an integral over this angle. After simple algebraic steps we obtain

$$
\begin{aligned}
& {\left[R_{\mathrm{II}-\mathrm{AA}}\left(v^{\prime}, \boldsymbol{\Omega}^{\prime} ; v, \boldsymbol{\Omega}\right)\right]_{i j}=\frac{2 L_{u}+1}{2 S+1} \sum_{K} \sum_{J_{u} J_{u}^{\prime}} \sum_{J_{\ell} J_{\ell}^{\prime}}(-1)^{J_{\ell}-J_{\ell}^{\prime}}} \\
& \times 3\left(2 J_{u}+1\right)\left(2 J_{u}^{\prime}+1\right)\left(2 J_{\ell}+1\right)\left(2 J_{\ell}^{\prime}+1\right) \\
& \times\left\{\begin{array}{ccc}
L_{u} & L_{\ell} & 1 \\
J_{\ell} & J_{u} & S
\end{array}\right\}\left\{\begin{array}{ccc}
L_{u} & L_{\ell} & 1 \\
J_{\ell} & J_{u}^{\prime} & S
\end{array}\right\}\left\{\begin{array}{ccc}
L_{u} & L_{\ell} & 1 \\
J_{\ell}^{\prime} & J_{u} & S
\end{array}\right\} \\
& \times\left\{\begin{array}{ccc}
L_{u} & L_{\ell} & 1 \\
J_{\ell}^{\prime} & J_{u}^{\prime} & S
\end{array}\right\}\left\{\begin{array}{ccc}
K & J_{u}^{\prime} & J_{u} \\
J_{\ell} & 1 & 1
\end{array}\right\}\left\{\begin{array}{ccc}
K & J_{u}^{\prime} & J_{u} \\
J_{\ell}^{\prime} & 1 & 1
\end{array}\right\} \\
& \times\left[P^{(K)}\left(\boldsymbol{\Omega}^{\prime}, \boldsymbol{\Omega}\right)\right]_{i j} \frac{1}{1+\epsilon^{\prime}+2 \pi \mathrm{i} v_{J_{u}^{\prime} J_{u}} / A\left(L_{u} \rightarrow L_{\ell}\right)} \\
& \times \frac{1}{2 \pi \Delta v_{\mathrm{D}}^{2}} \int_{0}^{\pi} \mathrm{d} \Theta \exp \left[-\frac{\left(v^{\prime}-v+v_{J_{\ell}^{\prime} J_{\ell}}\right)^{2}}{4 \Delta v_{\mathrm{D}}^{2} \sin ^{2}(\Theta / 2)}\right] \\
& \times \frac{1}{2}\left[W\left(\frac{a}{\cos (\Theta / 2)}, \frac{v_{J_{u} J_{\ell}}+v_{J_{u} J_{\ell}^{\prime}}^{\prime}}{2 \cos (\Theta / 2)}\right)\right. \\
& \left.+W\left(\frac{a}{\cos (\Theta / 2)}, \frac{v_{J_{u}^{\prime} J_{\ell}}+v_{J_{u}^{\prime} J_{\ell}^{\prime}}^{\prime}}{2 \cos (\Theta / 2)}\right)^{*}\right] \text {. }
\end{aligned}
$$

In the applications that will be shown in Sect. 7, the $R_{\mathrm{II}-\mathrm{AA}}$ redistribution matrix will be calculated by evaluating the integral over the scattering angle through a numerical quadrature. It should be observed that it is also possible to push the analytical calculations of this integral a little bit further (see Appendix B). However, the numerical computation of the integrals appearing in the ensuing expression is not more advantageous.

\subsection{Total redistribution matrix}

By analogy with the two-level atom case (see Sect. 2), and recalling that we are neglecting the depolarizing effect of collisions, we consider the total redistribution matrix

$[R]_{i j}=\alpha\left[R_{\mathrm{II}-\mathrm{AA}}\right]_{i j}+(1-\alpha)\left[R_{\mathrm{III}}\right]_{i j}$,

where the branching ratio $\alpha$ is defined as (cf. Eq. (10))

$\alpha=\frac{\Gamma_{\mathrm{R}}+\Gamma_{\mathrm{I}}}{\Gamma_{\mathrm{R}}+\Gamma_{\mathrm{I}}+\Gamma_{\mathrm{E}}}=\frac{A\left(L_{u} \rightarrow L_{\ell}\right)+C_{S}\left(L_{u} \rightarrow L_{\ell}\right)}{A\left(L_{u} \rightarrow L_{\ell}\right)+C_{S}\left(L_{u} \rightarrow L_{\ell}\right)+Q_{\mathrm{el}}}$.

The quantity $Q_{\text {el. }}$ is now the rate of collisions inducing transitions between magnetic sublevels pertaining either to the same $J$-level or to different $J$-levels of the same term, consistently with the fact that both kind of collisions contribute to redistribute the photon frequency during the scattering process (see Sect. 3.1).

In general, collisions inducing transitions between different $J$-levels of the same term can be due to electrons, protons, or neutral hydrogen atoms. Given the approximate way the effect of these collisions is accounted for in our approach, we calculate the quantity $\Gamma_{\mathrm{E}}$ taking only the contribution of collisions (elastic and weakly inelastic) with neutral hydrogen atoms into account. The impact on atomic polarization of weakly inelastic collisions with protons and electrons should be considered when modeling scattering polarization in hydrogen lines (e.g., Sahal-Bréchot et al. 1996). Neglecting the effect of these collisions is, however, a suitable approximation for the solar H I Ly- $\alpha$ line (e.g., Štěpán \& Trujillo Bueno 2011, and more references therein), and we expect it to be a good approximation also for the solar Ly- $\alpha$ line of He II (e.g., Trujillo Bueno et al. 2012; Belluzzi et al. 2012).

Although Eq. (36) is not the result of a self-consistent physical derivation, it seems reasonable to assume that the total redistribution matrix is given by such a linear combination of $R_{\mathrm{II}}$ and $R_{\mathrm{III}}$. The coherence fraction $\alpha$ represents the probability that the photon, after being absorbed, is re-emitted before the atom undergoes a collision that redistributes the photon's frequency, provided that it is re-emitted at all. When $\Gamma_{\mathrm{E}} \gg \Gamma_{\mathrm{R}}+\Gamma_{\mathrm{I}}$ (i.e., when the probability that the atom suffers an elastic or weakly inelastic collision before it de-excites is very high), then $\alpha$ goes to zero, and the total redistribution matrix reduces to $R_{\mathrm{III}}$. On the contrary, when $\Gamma_{\mathrm{E}}=0$ (i.e., when there are no elastic or weakly inelastic collisions), then scattering is coherent in the atom rest frame and the total redistribution matrix reduces to $R_{\mathrm{II}}$. It can also be verified that for the particular case of $S=0, L_{u}=J_{u}$ and $L_{\ell}=J_{\ell}$, the redistribution matrix of a two-level atom derived by Bommier (1997a), in the limit of $D^{(K)}=0$, is recovered.

As previously observed, given the low densities in the upper solar chromosphere, in the line core region of strong resonance lines, the contribution of the $R_{\mathrm{III}}$ redistribution matrix is expected to be much smaller than that of $R_{\mathrm{II}}$. Neglecting the depolarizing effect of elastic and weakly inelastic collisions with neutral hydrogen atoms should represent therefore a good approximation for modeling the core of strong resonance lines (see, for example, the work of Derouich et al. 2007, concerning the $\mathrm{H}$ and $\mathrm{K}$ lines of $\mathrm{Ca}$ II). This approximation, on the other hand, is more questionable for modeling the far wings of the same lines, which form deeper in the solar atmosphere.

Since the angular and frequency dependencies can be factorized, the $R_{\mathrm{II}-\mathrm{AA}}$ redistribution matrix can be written in the form

$$
\left[R_{\mathrm{II}-\mathrm{AA}}\left(v^{\prime}, \boldsymbol{\Omega}^{\prime} ; v, \boldsymbol{\Omega}\right)\right]_{i j}=\sum_{K} r_{\mathrm{II}-\mathrm{AA}}^{(K)}\left(v^{\prime}, v\right)\left[P^{(K)}\left(\boldsymbol{\Omega}^{\prime}, \boldsymbol{\Omega}\right)\right]_{i j},
$$

where the explicit expression of the quantity $r_{\mathrm{II}-\mathrm{AA}}^{(K)}\left(v^{\prime}, v\right)$ can be easily found by comparison with Eq. (35). Similarly, the $R_{\mathrm{III}}$ redistribution matrix can be written in the form

$$
\left[R_{\mathrm{III}}\left(v^{\prime}, \boldsymbol{\Omega}^{\prime} ; v, \boldsymbol{\Omega}\right)\right]_{i j}=\sum_{K} r_{\mathrm{III}}^{(K)}\left(v^{\prime}, v\right)\left[P^{(K)}\left(\boldsymbol{\Omega}^{\prime}, \boldsymbol{\Omega}\right)\right]_{i j},
$$

with

$r_{\text {III }}^{(K)}\left(v^{\prime}, v\right)=\psi^{(K)}(v) \varphi\left(v^{\prime}\right)$.

The explicit expression of the functions $\psi^{(K)}(v)$ can be easily found by comparison with Eq. (26). In particular, we observe 
that $\psi^{(0)}(v)=\varphi(v) /\left(1+\epsilon^{\prime}\right)$. Recalling Eq. (3), the total redistribution matrix defined by Eq. (36) can thus be written in the form

$$
\begin{aligned}
{\left[R\left(v^{\prime}, \boldsymbol{\Omega}^{\prime} ; v, \boldsymbol{\Omega}\right)\right]_{i j}=} & \sum_{K Q}(-1)^{Q} \mathcal{T}_{Q}^{K}(i, \boldsymbol{\Omega}) \mathcal{T}_{-Q}^{K}\left(j, \boldsymbol{\Omega}^{\prime}\right) \\
& \times\left[\alpha r_{\mathrm{II}-\mathrm{AA}}^{(K)}\left(v^{\prime}, v\right)+(1-\alpha) r_{\mathrm{III}}^{(K)}\left(v^{\prime}, v\right)\right]
\end{aligned}
$$

\section{Redistribution matrix for a two-level atom with hyperfine structure}

The $R_{\mathrm{II}}$ and $R_{\mathrm{III}}$ redistribution matrices presented in Sects. 3.1 and 3.2 , respectively, can also be applied for describing a two-level atom with hyperfine structure (HFS), provided that the following formal substitutions are performed:

$$
L \rightarrow J, \quad S \rightarrow I, \quad J \rightarrow F,
$$

where $I$ is the nuclear spin and $F$ is the total angular momentum (electronic + nuclear). The resulting $R_{\mathrm{II}}$ and $R_{\mathrm{III}}$ redistribution matrices describe a two-level atom with HFS, under the assumptions that the lower $F$-levels (i.e., the $F$-levels pertaining to the lower $J$-level) are infinitely sharp, and that the magnetic sublevels of the lower $F$-levels are evenly populated and no interference is present between them. These redistribution matrices account for quantum interference between pairs of magnetic sublevels pertaining either to the same $F$-level or to different $F$-levels ( $F$-state interference) of the upper $J$-level. As for the two-term atom case, they are valid in the absence of magnetic fields, and they do not account for stimulated emission and for the depolarizing effect of elastic collisions. The $R_{\mathrm{III}}$ redistribution matrix describes scattering processes in the limit in which collisions are extremely efficient in inducing transitions between magnetic sublevels pertaining either to the same $F$-level or to different $F$-levels of the upper $J$-level. Since the energy splitting of HFS levels is typically much smaller than that of fine structure levels, this limit results to be justified for physical conditions that are less restrictive than for the case of a two-term atom (see Sect. 3.1).

Also for this atomic model, in the absence of a selfconsistent PRD theory, we assume that the most general redistribution matrix is given by the linear combination $\alpha R_{\mathrm{II}}+(1-\alpha) R_{\mathrm{III}}$. The branching ratio $\alpha$ is given by:

$\alpha=\frac{\Gamma_{\mathrm{R}}+\Gamma_{\mathrm{I}}}{\Gamma_{\mathrm{R}}+\Gamma_{\mathrm{I}}+\Gamma_{\mathrm{E}}}=\frac{A\left(J_{u} \rightarrow J_{\ell}\right)+C_{S}\left(J_{u} \rightarrow J_{\ell}\right)}{A\left(J_{u} \rightarrow J_{\ell}\right)+C_{S}\left(J_{u} \rightarrow J_{\ell}\right)+Q_{\mathrm{el}} .}$,

where $Q_{\mathrm{el}}$ is the rate of collisions (with neutral hydrogen atoms) inducing transitions between magnetic sublevels pertaining either to the same $F$-level or to different $F$-levels of the same $J$-level.

\section{Radiative transfer equations}

We consider a plane-parallel atmosphere in the absence of magnetic fields. The problem is thus characterized by cylindrical symmetry along the direction perpendicular to the surface of the atmosphere (hereafter, the "vertical"). Taking the quantization axis directed along the vertical, and the reference direction for positive $Q$ parallel to the surface, at any height in the atmosphere the radiation field is only described by the Stokes parameters $I$ and $Q$, while $J_{0}^{0}$ and $J_{0}^{2}$ are the only non-vanishing elements of the radiation field tensor. Under the above-mentioned hypotheses, and neglecting lower term polarization and stimulated emission, the radiative transfer equation takes the simplified form

$\frac{\mathrm{d}}{\mathrm{d} s}\left(\begin{array}{c}I(v, \boldsymbol{\Omega}) \\ Q(v, \boldsymbol{\Omega})\end{array}\right)=-\eta_{I}(v)\left(\begin{array}{c}I(v, \boldsymbol{\Omega}) \\ Q(v, \boldsymbol{\Omega})\end{array}\right)+\left(\begin{array}{c}\varepsilon_{I}(v, \boldsymbol{\Omega}) \\ \varepsilon_{Q}(v, \boldsymbol{\Omega})\end{array}\right)$,

where $s$ is the coordinate measured along the ray path, and $\eta_{I}(v)$ is the intensity absorption coefficient. The emission and absorption coefficients appearing in Eq. (44) contain both the line and the continuum contributions (hereafter indicated with the superscripts $\ell$ and $c$, respectively). Introducing the optical depth $\tau_{v}$ defined by

$\mathrm{d} \tau_{v}=-\eta_{I}(v) \mathrm{d} s$,

and assuming that the continuum is unpolarized, the radiative transfer equation takes the form

$\frac{\mathrm{d}}{\mathrm{d} \tau_{v}}\left(\begin{array}{c}I(v, \boldsymbol{\Omega}) \\ Q(v, \boldsymbol{\Omega})\end{array}\right)=\left(\begin{array}{c}I(v, \boldsymbol{\Omega}) \\ Q(v, \boldsymbol{\Omega})\end{array}\right)-\left(\begin{array}{c}S_{I}(v, \boldsymbol{\Omega}) \\ S_{Q}(v, \boldsymbol{\Omega})\end{array}\right)$,

where the source functions are given by

$S_{I}(v, \boldsymbol{\Omega})=\frac{\varepsilon_{I}(v, \boldsymbol{\Omega})}{\eta_{I}(v)}=\frac{\varepsilon_{I}^{\ell}(v, \boldsymbol{\Omega})+\varepsilon_{I}^{\mathrm{c}}(v)}{\eta_{I}^{\ell}(v)+\eta_{I}^{\mathrm{c}}(v)}$,
$S_{Q}(v, \boldsymbol{\Omega})=\frac{\varepsilon_{Q}(v, \boldsymbol{\Omega})}{\eta_{I}(v)}=\frac{\varepsilon_{Q}^{\ell}(v, \boldsymbol{\Omega})}{\eta_{I}^{\ell}(v)+\eta_{I}^{\mathrm{c}}(v)}$.

Introducing the line and continuum source functions

$S_{I}^{\ell}=\frac{\varepsilon_{I}^{\ell}}{\eta_{I}^{\ell}}, \quad S_{Q}^{\ell}=\frac{\varepsilon_{Q}^{\ell}}{\eta_{I}^{\ell}}, \quad S_{I}^{\mathrm{c}}=\frac{\varepsilon_{I}^{\mathrm{c}}}{\eta_{I}^{\mathrm{c}}}$,

and the quantity

$r_{v}=\frac{\eta_{I}^{\ell}(v)}{\eta_{I}^{\ell}(v)+\eta_{I}^{\mathrm{c}}(v)}$

we have

$S_{I}(v, \boldsymbol{\Omega})=r_{v} S_{I}^{\ell}(v, \boldsymbol{\Omega})+\left(1-r_{v}\right) S_{I}^{\mathrm{c}}(v)$,

$S_{Q}(\nu, \boldsymbol{\Omega})=r_{v} S_{Q}^{\ell}(\nu, \boldsymbol{\Omega})$.

\subsection{Line source function}

The line absorption coefficient $\eta_{I}^{\ell}(v)$ is given by

$\eta_{I}^{\ell}(v)=k_{L} \varphi(v)$

where the frequency-integrated absorption coefficient, $k_{L}$, and the normalized absorption profile for the multiplet, $\varphi(v)$, are defined by Eqs. (14) and (23), respectively. The line emission coefficient is given by

$$
\begin{aligned}
\varepsilon_{i}^{\ell}(v, \boldsymbol{\Omega})= & k_{L} \int \mathrm{d} v^{\prime} \oint \frac{\mathrm{d} \mathbf{\Omega}^{\prime}}{4 \pi} \sum_{j=0}^{3}\left[R\left(v^{\prime}, \mathbf{\Omega}^{\prime} ; v, \boldsymbol{\Omega}\right)\right]_{i j} I_{j}\left(v^{\prime}, \mathbf{\Omega}^{\prime}\right) \\
& +\frac{\epsilon^{\prime}}{1+\epsilon^{\prime}} k_{L} \varphi(v) B_{T}\left(v_{0}\right) \delta_{i 0},
\end{aligned}
$$

where $R\left(v^{\prime}, \boldsymbol{\Omega}^{\prime} ; v, \boldsymbol{\Omega}\right)$ is the redistribution matrix defined in Eq. (41). Substituting Eq. (41) into Eq. (54), and recalling 
the definition of the monochromatic radiation field tensor (see Eq. (22)), the line emission coefficient can be written as

$$
\begin{aligned}
& \varepsilon_{i}^{\ell}(v, \boldsymbol{\Omega})=k_{L} \sum_{K Q}(-1)^{Q} \mathcal{T}_{Q}^{K}(i, \boldsymbol{\Omega}) \\
& \quad \times \int \mathrm{d} v^{\prime} J_{-Q}^{K}\left(v^{\prime}\right)\left[\alpha r_{\mathrm{II}-\mathrm{AA}}^{(K)}\left(v^{\prime}, v\right)+(1-\alpha) r_{\mathrm{III}}^{(K)}\left(v^{\prime}, v\right)\right] \\
& \quad+\frac{\epsilon^{\prime}}{1+\epsilon^{\prime}} k_{L} \varphi(v) B_{T}\left(v_{0}\right) \delta_{i 0} .
\end{aligned}
$$

Introducing the quantity

$\tilde{J}_{Q}^{K}(v)=\int \mathrm{d} v^{\prime} J_{Q}^{K}\left(v^{\prime}\right) g^{(K)}\left(v^{\prime}, v\right)$,

with

$g^{(K)}\left(v^{\prime}, v\right)=\left[\alpha \frac{r_{\mathrm{II}-\mathrm{AA}}^{(K)}\left(v^{\prime}, v\right)}{\varphi(v)}+(1-\alpha) \frac{r_{\mathrm{III}}^{(K)}\left(v^{\prime}, v\right)}{\varphi(v)}\right]$,

the line source function can be written as

$S_{i}^{\ell}(v, \boldsymbol{\Omega})=\frac{\varepsilon_{i}^{\ell}(v, \boldsymbol{\Omega})}{\eta_{I}^{\ell}(v)}=\sum_{K Q}(-1)^{Q} \mathcal{T}_{Q}^{K}(i, \boldsymbol{\Omega}) S_{Q}^{K}(v)$,

where we have introduced the multipole components of the line source function, defined by

$S_{Q}^{K}(v)=\tilde{J}_{-Q}^{K}(v)+\frac{\epsilon^{\prime}}{1+\epsilon^{\prime}} B_{T}\left(v_{0}\right) \delta_{K 0} \delta_{Q 0}$.

Recalling Eq. (40), and recalling the definition of the frequencyintegrated radiation field tensor, $\bar{J}_{Q}^{K}$ (see Eq. (24)), the quantity $\tilde{J}_{Q}^{K}(v)$ can be written as

$\tilde{J}_{Q}^{K}(v)=\alpha \int \mathrm{d} v^{\prime} J_{Q}^{K}\left(v^{\prime}\right) g_{\mathrm{II}-\mathrm{AA}}^{(K)}\left(v^{\prime}, v\right)+(1-\alpha) \frac{\psi^{(K)}(v)}{\varphi(v)} \bar{J}_{Q}^{K}$,

with

$g_{\mathrm{II}-\mathrm{AA}}^{(K)}\left(v^{\prime}, v\right) \equiv \frac{r_{\mathrm{II}-\mathrm{AA}}^{(K)}\left(v^{\prime}, v\right)}{\varphi(v)}$.

An analysis of the functions $g_{\mathrm{II}-\mathrm{AA}}^{(K)}\left(v^{\prime}, v\right)$ is carried out in Appendix C. Due to the peaked shape of these functions, the numerical evaluation of the integral in the righthand side of Eq. (60) (often referred to as the scattering integral) is not trivial, and indeed has already received some attention in the past for the unpolarized case (e.g., Adams et al. 1971; Gouttebroze 1986; Uitenbroek 1989). The numerical techniques used in this work for the calculation of this integral are discussed in some detail in Appendix D.

\subsection{Continuum source function}

We consider an unpolarized continuum, characterized by the total absorption coefficient (opacity)

$\eta_{I}^{\mathrm{c}}(v)=k_{\mathrm{c}}(v)+\sigma(v)$,

where $\sigma$ is the continuum scattering coefficient, and $k_{\mathrm{c}}$ is the continuum true absorption coefficient. As far as the continuum emission coefficient is concerned, we distinguish between the thermal term, $b(v)$, and the scattering term. Since in the atmospheric layers of interest the anisotropy degree of the solar radiation field is very small, in the scattering term we can safely neglect the contribution of the multipole components of the radiation field tensor $J_{Q}^{K}(v)$ with $K \neq 0$, and we can write

$\varepsilon_{I}^{\mathrm{c}}(v)=b(v)+\sigma(v) J_{0}^{0}(v)$

where we recall that the 0-rank element of the radiation field tensor, $J_{0}^{0}$, represents the radiation field averaged over the solid angle. The continuum source function is thus given by

$S_{I}^{\mathrm{c}}(v)=\frac{b(v)}{k_{\mathrm{c}}(v)+\sigma(v)}+\frac{\sigma(v)}{k_{\mathrm{c}}(v)+\sigma(v)} J_{0}^{0}(v)$.

\subsection{Formal solution of the radiative transfer equations}

The calculation of $J_{0}^{0}(v)$ and $J_{0}^{2}(v)$ at each height in the atmosphere requires the knowledge of the Stokes parameters $I(v, \mathbf{\Omega})$ and $Q(v, \mathbf{\Omega})$ of the radiation propagating along the directions of the chosen angular quadrature, as obtained from the solution of the radiative transfer equation. Due to the symmetry of the problem, the radiation field only depends on the inclination $\theta$ with respect to the vertical. Hereafter we will thus indicate the Stokes parameters with the notation $I_{i}(v, \mu ; h)$ with $i=0,1$ (standing for $I$ and $Q$, respectively), $\mu=\cos \theta$, and with $h$ the height in the atmosphere. The radiative transfer equations for $I$ and $Q$ are formally identical and completely decoupled (see Eq. (46)). Their formal solution is given by

$I_{i}(v, \mu ; \mathrm{O})=I_{i}(v, \mu ; \mathrm{M}) \mathrm{e}^{-\Delta \tau_{v}}+\int_{0}^{\Delta \tau_{v}} S_{i}(\nu, \mu ; t) \mathrm{e}^{-t} \mathrm{~d} t$,

where $I_{i}(v, \mu ; \mathrm{O})$ are the Stokes parameters of the radiation of frequency $v$, at point $\mathrm{O}$, propagating along the direction $\mu, \mathrm{M}$ is the upwind point in the given geometrical discretization of the atmosphere, $\Delta \tau_{v}$ is the optical distance (measured along the ray path) between points $\mathrm{O}$ and $\mathrm{M}$, at frequency $v$, and $S_{i}(\nu, \mu ; t)$ are the source functions in $I$ and $Q$, defined by Eqs. (47) and (48), respectively, at point of optical depth $t$.

We evaluate the integral in the righthand side of Eq. (65) by means of the short-characteristic method (see Kunasz \& Auer 1988):

$$
\begin{aligned}
\int_{0}^{\Delta \tau_{v}} & S_{i}(\nu, \mu ; t) \mathrm{e}^{-t} \mathrm{~d} t= \\
& \Psi_{\mathrm{M}} S_{i}(\nu, \mu ; \mathrm{M})+\Psi_{\mathrm{O}} S_{i}(\nu, \mu ; \mathrm{O})+\Psi_{\mathrm{P}} S_{i}(\nu, \mu ; \mathrm{P}),
\end{aligned}
$$

where $\mathrm{P}$ is the downwind point, and where $\Psi$ are coefficients depending on the optical distances between points $\mathrm{M}$ and $\mathrm{O}$, and between points $\mathrm{O}$ and P. Indicating the values of the $I$ and $Q$ Stokes parameters and of the corresponding source functions at the various points of the spatial grid used for the discretization of the atmosphere through the elements of column vectors, the formal solution of the radiative transfer equation can be written in the form

$\boldsymbol{I}_{i}(v, \mu)=\boldsymbol{\Lambda}_{v \mu} \boldsymbol{S}_{i}(v, \mu)+\boldsymbol{T}_{i}(v, \mu)$,

where $\boldsymbol{T}_{i}(\nu, \mu)$ are the transmitted $I$ and $Q$ Stokes parameters due to the incident radiation at the boundary, and $\boldsymbol{\Lambda}_{v \mu}$ is a $N P \times N P$ operator, with $N P$ the number of points of the spatial grid. Equivalently, we can write

$I_{i}(v, \mu ; \ell)=\sum_{m=1}^{N P} \Lambda_{\nu \mu}(\ell, m) S_{i}(\nu, \mu ; m)+T_{i}(\nu, \mu ; \ell)$,

with $\ell, m=1, \ldots, N P$. We recall that the element $\Lambda_{\nu \mu}(\ell, \ell)$ represents the specific intensity of the radiation of frequency $v$, 
at point $\ell$, propagating in direction $\mu$, as obtained assuming that there is no transmitted radiation from the boundaries, and that the intensity source function is everywhere zero, except at point $\ell$ where it is equal to 1 .

Substituting Eq. (68) into Eq. (22) and the ensuing equation into Eq. (56), recalling the expressions of the line and continuum source functions previously derived, the operations required for the numerical calculation of $\tilde{J}_{0}^{K}(v)$ at point $\ell$ can be indicated as follows:

$$
\begin{aligned}
\tilde{J}_{0}^{K}(v ; \ell)= & \int \mathrm{d} v^{\prime} g^{(K)}\left(v^{\prime}, v ; \ell\right) \\
& \times\left\{\sum _ { m = 1 } ^ { N P } \left[r_{v^{\prime}}(m) \sum_{K^{\prime}} \Lambda_{K 0, K^{\prime} 0}\left(v^{\prime} ; \ell, m\right) S_{0}^{K^{\prime}}\left(v^{\prime} ; m\right)\right.\right. \\
& \left.\left.+\left(1-r_{v^{\prime}}(m)\right) \Lambda_{K 0}^{\mathrm{c}}\left(v^{\prime} ; \ell, m\right) S_{I}^{\mathrm{c}}\left(v^{\prime} ; m\right)\right]+T_{0}^{K}\left(v^{\prime} ; \ell\right)\right\}
\end{aligned}
$$

where we have explicitly indicated the dependence on the height point in the atmosphere of the various physical quantities previously introduced. The $\Lambda$ operators and the $T_{Q}^{K}$ tensors are given by ${ }^{7}$

$$
\begin{aligned}
& \Lambda_{K Q, K^{\prime} Q^{\prime}}(v ; \ell, m)=\int \frac{\mathrm{d} \boldsymbol{\Omega}}{4 \pi} \sum_{i=0}^{3}(-1)^{Q^{\prime}} \mathcal{T}_{Q}^{K}(i, \boldsymbol{\Omega}) \mathcal{T}_{Q^{\prime}}^{K^{\prime}}(i, \boldsymbol{\Omega}) \\
& \times \Lambda_{v \mu}(\ell, m), \\
& T_{Q}^{K}(v ; \ell)=\int \frac{\mathrm{d} \boldsymbol{\Omega}}{4 \pi} \sum_{i=0}^{3} \mathcal{T}_{Q}^{K}(i, \boldsymbol{\Omega}) T_{i}(v, \mu ; \ell) .
\end{aligned}
$$

Equation (69) is the generalization to the PRD case of Eqs. (17) and (18) of Trujillo Bueno \& Manso Sainz (1999). The operators defined by Eqs. (70) and (71) are analogous to those defined in Eqs. (19)-(24) of Trujillo Bueno \& Manso Sainz (1999); the main difference is that in the PRD case the integral over the frequency of the incoming radiation $v^{\prime}$ appearing in Eq. (69) cannot be included in the definition of these operators since also the source function now depends on $v^{\prime}$.

\section{Iterative solution of the non-LTE problem}

The equations for $S_{0}^{0}$ and $S_{0}^{2}$ resulting from substitution of Eq. (69) into Eq. (59) represent the fundamental equations for the non-LTE problem under consideration. It is well known that the most suitable approach for the numerical solution of this set of equations is through iterative methods. In this work, we apply the Jacobian-based iterative method described below.

Let $S_{0}^{0 \text { old }}$ and $S_{0}^{2 \text { old }}$ be given estimates of the unknowns at the various points of the grid. The lambda iteration method consists in calculating $\tilde{J}_{0}^{0}$ and $\tilde{J}_{0}^{2}$ through Eq. (69) by using these "old" values of the source function, and in substituting the ensuing values into Eq. (59) in order to find the new estimates $S_{0}^{0 \text { new }}$ and $S_{0}^{2 \text { new }}$. This is actually the simplest iterative method, but its convergence rate in optically thick media is, as known,

\footnotetext{
7 Note that the definition of the operators $\Lambda_{K Q, K^{\prime} Q^{\prime}}$ given in Eq. (70) is very general, being valid for arbitrary geometries. In the particular case we are considering here, in which $Q=Q^{\prime}=0$ and $K, K^{\prime}=0,2$, the sum over $i$ can be limited to $i=0,1$. This restriction on the index $i$ also holds for Eq. (72).
}

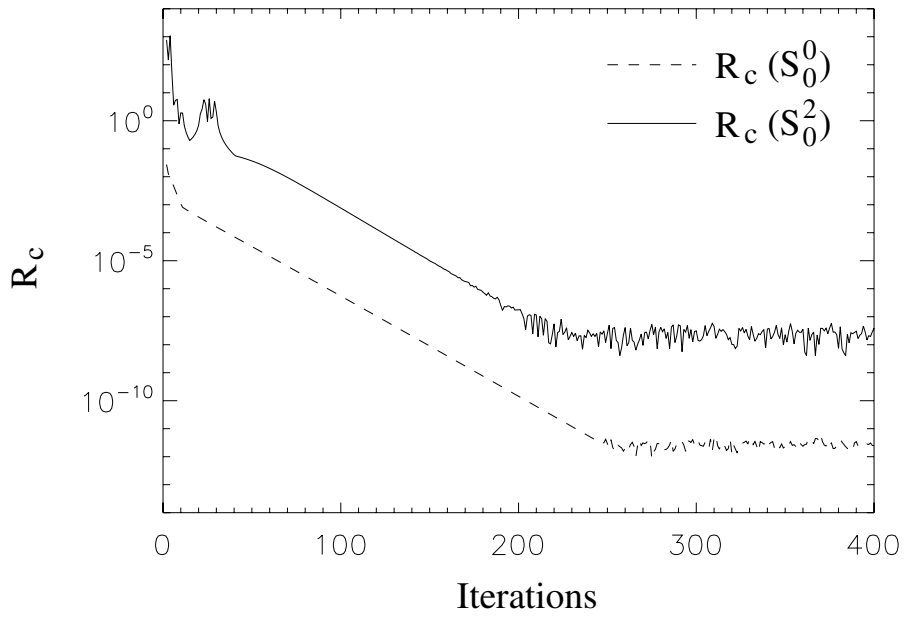

Fig. 1. Variation with the iteration number of the maximum relative change, $R_{\mathrm{c}}$ (see Eq. (77)), of the multipole components of the line source function $S_{0}^{0}$ (dashed line) and $S_{0}^{2}$ (solid line).

extremely slow. The Jacobian iterative method consists in calculating $\tilde{J}_{0}^{0}$ and $\tilde{J}_{0}^{2}$ at any grid point $\ell$ through Eq. (69) by using the "old" values of the source function at all the grid points, except at point $\ell$ where the new estimates, $S_{0}^{0 \text { new }}$ and $S_{0}^{2 \text { new }}$, are implicitly (their value being still unknown) used. The new values of the source function are then obtained by substituting the ensuing expressions of $\tilde{J}_{0}^{0}$ and $\tilde{J}_{0}^{2}$ into Eq. (59). In formulae, we have

$$
\begin{aligned}
\tilde{J}_{0}^{K}(v ; \ell)= & \tilde{J}_{0}^{K}(v ; \ell)^{\text {old }}+\int \mathrm{d} v^{\prime} g^{(K)}\left(v^{\prime}, v ; \ell\right) r_{v^{\prime}}(\ell) \\
& \times \sum_{K^{\prime}} \Lambda_{K 0, K^{\prime} 0}\left(v^{\prime} ; \ell, \ell\right) \Delta S_{0}^{K^{\prime}}\left(v^{\prime} ; \ell\right),
\end{aligned}
$$

where

$\Delta S_{Q}^{K}\left(v^{\prime} ; \ell\right)=S_{Q}^{K}\left(v^{\prime} ; \ell\right)^{\text {new }}-S_{Q}^{K}\left(v^{\prime} ; \ell\right)^{\text {old }}$,

and where $\tilde{J}_{0}^{0 \text { old }}$ and $\tilde{J}_{0}^{2 \text { old }}$ are the values of $\tilde{J}_{0}^{0}$ and $\tilde{J}_{0}^{2}$ that are obtained from a formal solution of the radiative transfer equation, carried out using the "old" estimates of $S_{0}^{0}$ and $S_{0}^{2}$. Equation (73) is analogous to Eqs. (25) and (26) of Trujillo Bueno \& Manso Sainz (1999).

The Jacobi-based method that we apply in this work is obtained by setting $\Lambda_{K 0, K^{\prime} 0}=\Lambda_{00,00} \delta_{K 0} \delta_{K^{\prime} 0}$ (see Trujillo Bueno \& Manso Sainz 1999, for a detailed discussion on the role of the various $\Lambda$-operators). Introducing the ensuing expressions into Eq. (59) we obtain

$$
\begin{aligned}
\Delta S_{0}^{0}(v ; \ell)= & \int \mathrm{d} v^{\prime} g^{(0)}\left(v^{\prime}, v ; \ell\right) r_{v^{\prime}}(\ell) \Lambda_{00,00}\left(v^{\prime} ; \ell, \ell\right) \Delta S_{0}^{0}\left(v^{\prime} ; \ell\right) \\
& +\tilde{J}_{0}^{0}(v ; \ell)^{\text {old }}+\frac{\epsilon^{\prime}}{1+\epsilon^{\prime}} B_{T}(v)-S_{0}^{0}(v ; \ell)^{\text {old }} \\
\Delta S_{0}^{2}(v ; \ell)= & \tilde{J}_{0}^{2}(v ; \ell)^{\text {old }}-S_{0}^{2}(v ; \ell)^{\text {old }}
\end{aligned}
$$

We observe that the Jacobi-based method here applied provides a new estimate of $S_{0}^{2}$ that is formally identical to that given by the $\Lambda$-iteration method. There is however an important difference in that the quantity $\tilde{J}_{0}^{2}$ (which is dominated by Stokes $I$, which in turn is set by $S_{0}^{0}$ ) is in this case improved at the rate given by the equation that yields $S_{0}^{0}$ (see Trujillo Bueno $\&$ Manso Sainz 1999). We calculate the new estimate of $S_{0}^{0}$ by solving Eq. (75) through the "Frequency-By-Frequency" (FBF) method described in Appendix E. 
L. Belluzzi and J. Trujillo Bueno: The transfer of resonance line polarization with PRD and $J$-state interference
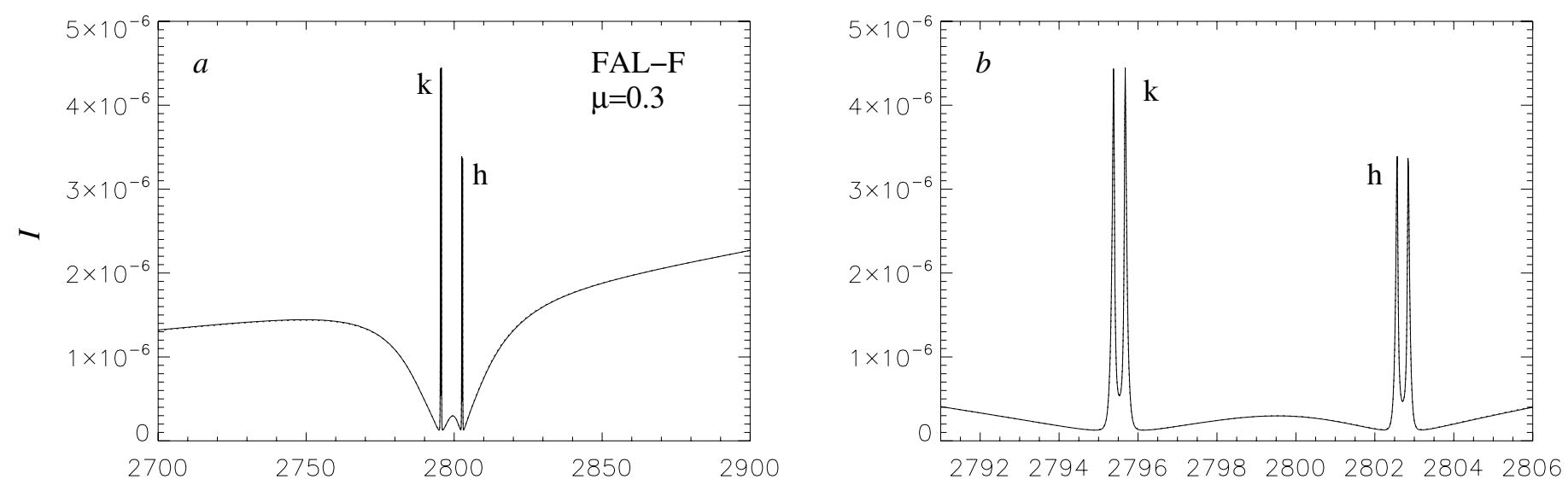

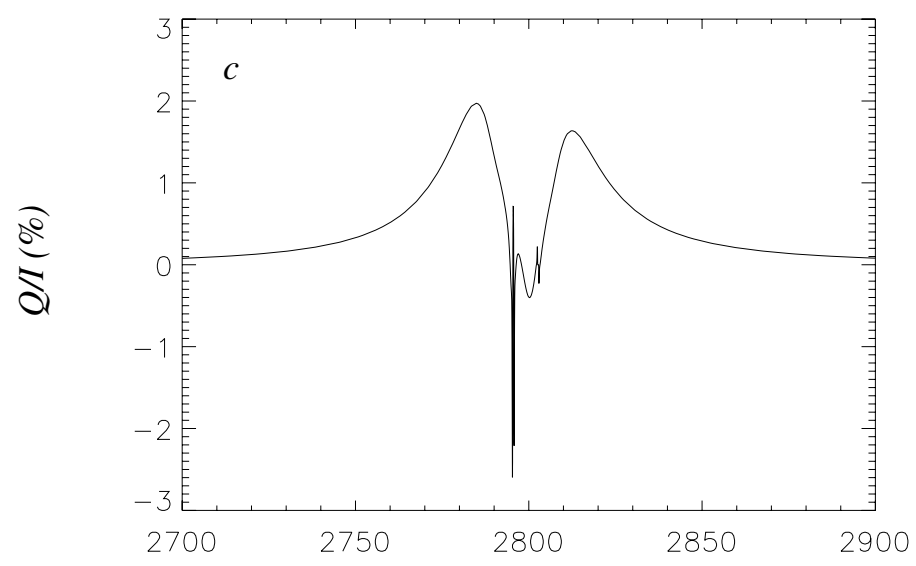

$\lambda(\AA)$

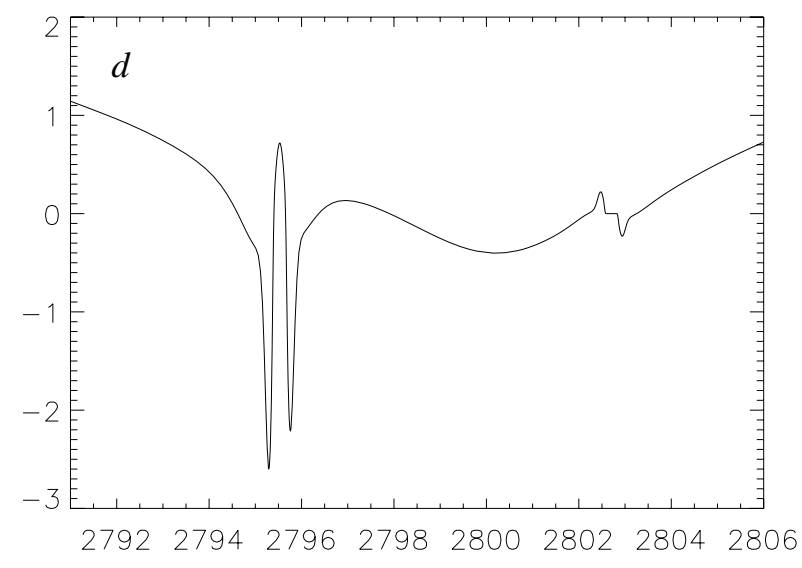

$\lambda(\AA)$

Fig. 2. Stokes $I$ and $Q / I$ profiles of the $\mathrm{Mg}$ II h and $\mathrm{k}$ lines calculated in the FAL-F atmospheric model taking PRD and $J$-state interference into account. Panel a) intensity profile of the radiation emergent at $\mu=0.3$ across the two lines (expressed in erg cm${ }^{-2} \mathrm{~s}^{-1} \mathrm{~Hz}^{-1} \mathrm{sr}^{-1}$ ). The intensity profile calculated by Uitenbroek's (2001) code is overplotted with dotted line, but it cannot be distinguished from that obtained with our two-term atom polarization transfer code. Panel b) same as panel a), but for a smaller wavelength interval in order to better visualize the line-core region of the two lines. Panel c) $Q / I$ profile. The reference direction for positive $Q$ is the parallel to the nearest limb. Panel d) same as panel c) but for a smaller wavelength interval.

\section{Illustrative application}

In forthcoming publications, we will apply the theoretical approach and the numerical techniques described in this paper to the investigation of strong chromospheric lines of particular interest. Here we only show an illustrative application to the $\mathrm{Mg} \mathrm{II} \mathrm{h}$ and $\mathrm{k}$ lines, for the solar atmospheric model $\mathrm{F}$ of Fontenla et al. (1993). We first solve the problem for the unpolarized case by applying the radiative transfer code by Uitenbroek (2001). The values of $J_{0}^{0}$ and $J_{0}^{2}$ corresponding to the converged solution of the unpolarized problem are the initial guess for our iterative method. We consider a two-term $\left({ }^{2} S-{ }^{2} P\right)$ model atom for $\mathrm{Mg}$ II. The lower term is composed of the ground level $\left({ }^{2} S_{1 / 2}\right)$, while the upper term is composed of the upper levels of the $\mathrm{h}$ and $\mathrm{k}$ lines $\left({ }^{2} P_{1 / 2}\right.$ and ${ }^{2} P_{3 / 2}$, respectively). The lower level population is read from the output of Uitenbroek's code, and it is not recalculated. Also the continuum opacity and thermal emission, as well as the elastic and inelastic collisional rates are read from the output of Uitenbroek's code. A very high angular resolution is used in the calculations. In particular, we apply a Gauss-Legendre quadrature, with 20 values of $\mu$ in the interval $[-1,0]$ (incoming radiation), and 20 values of $\mu$ in the interval $[0,1]$ (outgoing radiation). The convergence rate of the iterative method in this particular problem, expressed through the maximum relative change

$R_{\mathrm{c}}\left(S_{0}^{K}\right)=\max \left(\frac{\left|S_{0}^{K}(v ; \ell)^{\text {new }}-S_{0}^{K}(v ; \ell)^{\text {old }}\right|}{\left|S_{0}^{K}(v ; \ell)^{\text {new }}\right|}\right)$,

where $K=0,2$, and where the maximum is evaluated over all frequencies and atmospheric heights, is shown in Fig. 1. As expected, the iterative method converges at the rate of the Jacobi iterations (cf. Trujillo Bueno \& Manso Sainz 1999).

Intensity and $Q / I$ profiles of the radiation emergent at $\mu=$ 0.3 are shown in Fig. 2. The PRD intensity profiles that result from the application of our two-term atom polarized radiative transfer code are identical to those calculated by Uitenbroek's (2001) PRD code (which does not take scattering polarization and $J$-state interference into account). As shown in the lower panels of Fig. 2, the $Q / I$ profile is characterized by a complex pattern, with large positive polarization amplitudes in the blue and red wings and a negative minimum in the spectral region between the $\mathrm{k}$ and $\mathrm{h}$ lines. Moreover, it shows a conspicuous nearly symmetric triplet peak polarization structure around the core of the $\mathrm{k}$ line and an antisymmetric signal around the h-line center (cf. Belluzzi \& Trujillo Bueno 2012). 


\section{Conclusions}

Information on the magnetism of the outer atmosphere of the Sun and of other stars is encoded in the polarization that several physical mechanisms introduce in spectral lines. Of particular interest is the modification, due to the Hanle effect induced by weak $(B \lesssim 100 \mathrm{G})$ magnetic fields, of the linear polarization signals produced by scattering processes in strong resonance lines. In order to model correctly the spectral details of scattering polarization signals generated in strong resonance lines in an optically thick plasma, it is essential to solve the ensuing non-LTE radiative transfer problem taking PRD and $J$-state interference effects into account. In this paper we have presented a theoretical approach to this complex problem, as well as the numerical methods for the solution of the ensuing equations. This first investigation has been restricted to the unmagnetized reference case.

We have considered a two-term atom with unpolarized lower term and infinitely sharp lower levels. This atomic model accounts for quantum interference between sublevels pertaining either to the same $J$-level or to different $J$-levels of the upper term, and it is suitable for modeling several resonance lines of high diagnostic interest (e.g., $\mathrm{Mg}$ II $\mathrm{h}$ and $\mathrm{k}, \mathrm{H}$ I Ly- $\alpha$, He II Ly- $\alpha$ ). By analogy with the two-level atom case, we have assumed that the redistribution matrix for such an atomic model is given by the linear combination of two terms, one describing coherent scattering processes in the atom rest frame $\left(R_{\mathrm{II}}\right)$, and one describing scattering processes in the limit of complete frequency redistribution $\left(R_{\mathrm{III}}\right)$. The expression of $R_{\mathrm{II}}$ has been derived within the framework of the theoretical approach presented in Landi Degl'Innocenti et al. (1997) (suitable for treating the limit of coherent scattering), while the expression of $R_{\mathrm{III}}$ has been deduced within the framework of the theory of polarization described in LL04 (suitable for treating the limit of CRD).

Concerning the $R_{\mathrm{III}}$ redistribution matrix, we have derived an approximate expression that describes scattering events in the limit in which collisions are able to redistribute the photon frequency across the whole multiplet. As discussed in Sect. 3.1, this is in general a strong assumption, especially when the energy separation among the various $J$-levels is very large. This assumption is ultimately due to the fact that our $R_{\mathrm{III}}$ has been derived in a heuristic way starting from the theory of LL04, which is based on the flat-spectrum approximation. Although other approximate forms of $R_{\mathrm{III}}$, based on different hypotheses, can in principle be proposed (see, for example, Smitha et al. 2013), we believe that the theory of LL04 is at the moment the most robust one for deriving this redistribution matrix. A comparison between the different expressions of $R_{\mathrm{III}}$ that have been proposed up to now, and a detailed analysis of their properties and weaknesses will be the object of a forthcoming paper.

The depolarizing effect of elastic and weakly inelastic collisions has been neglected. This is not fully consistent, but accounting for this effect gives rise to a series of difficulties that go beyond the scope of this work. As discussed in Sect. 3.3, this should be in any case a suitable approximation for modeling the line-core signal of strong resonance lines forming in the upper chromosphere and transition region of the Sun.

As pointed out in Sect. 4, performing a series of formal substitutions of the quantum numbers, the redistribution matrices presented in this work for a two-term atom can be applied to describe a two-level atom with HFS (i.e., a model atom accounting for interference between pairs of magnetic sublevels pertaining either to the same HFS $F$-level, or to different $F$-levels of the same $J$-level). The hypotheses under which the physical scenario described by our $R_{\text {III }}$ redistribution matrix is strictly justified turn out to be less restrictive in a two-level atom with HFS than in a two-term atom.

The numerical method of solution we have developed for solving this non-LTE radiative transfer problem is based on a direct generalization of the Jacobian iterative scheme developed by Trujillo Bueno \& Manso Sainz (1999) for the case of a two-level atom (i.e., without $J$-state interference), in the limit of CRD. The ensuing computer program can be applied to investigate a number of interesting radiative transfer problems in solar and stellar physics (e.g., Belluzzi \& Trujillo Bueno 2012; Belluzzi et al. 2012; Belluzzi \& Trujillo Bueno 2013). Moreover, it can be used as starting point for further developments, such as the modeling of the Hanle effect and/or the investigation of the scattering polarization that results from the interaction between the line and continuum processes. We plan to address several of these issues in forthcoming papers.

Acknowledgements. We wish to thank Véronique Bommier (Observatoire de Paris) for several useful comments and suggestions that allowed us to deepen and clarify a series of critical aspects of the PRD problem. The authors are also very grateful to Egidio Landi Degl'Innocenti (University of Florence) for several clarifying discussions on the theoretical approaches that have been used as starting point for our work. Financial support by the Spanish Ministry of Economy and Competitiveness and the European FEDER Fund through projects AYA2010-18029 (Solar Magnetism and Astrophysical Spectropolarimetry) and CONSOLIDER INGENIO CSD2009-00038 (Molecular Astrophysics: The Herschel and Alma Era) is gratefully acknowledged.

\section{Appendix A: Derivation of the $R_{\| \mid}$redistribution function in the observer's frame}

Let us consider an atom moving with velocity $\boldsymbol{v}$ in the observer's frame. Let $v^{\prime}$ and $v$ be the frequencies of the incoming and outgoing photons, respectively, in the observer's frame, and let $\xi^{\prime}$ and $\xi$ be the corresponding frequencies in the atom rest frame. Taking the Doppler effect to first order in $v / c$ into account, and neglecting aberration effects, we have

$$
\begin{aligned}
\xi & =v-\frac{v_{0}}{c} \boldsymbol{v} \cdot \boldsymbol{\Omega}, \\
\xi^{\prime} & =v^{\prime}-\frac{v_{0}}{c} \boldsymbol{v} \cdot \boldsymbol{\Omega}^{\prime},
\end{aligned}
$$

where $\boldsymbol{\Omega}^{\prime}$ and $\boldsymbol{\Omega}$ are the progation directions of the incoming and outgoing photons, respectively. Recalling Eq. (30), the redistribution matrix, in the observer's frame, for the atom under consideration is given by

$$
\begin{aligned}
& {\left[R_{\mathrm{II}}\left(v^{\prime}, \boldsymbol{\Omega}^{\prime} ; v, \boldsymbol{\Omega} ; \boldsymbol{v}\right)\right]_{i j}=\frac{2 L_{u}+1}{2 S+1} \sum_{K} \sum_{J_{u} J_{u}^{\prime}} \sum_{J_{\ell} J_{\ell}^{\prime}}(-1)^{J_{\ell}-J_{\ell}^{\prime}}} \\
& \quad \times 3\left(2 J_{u}+1\right)\left(2 J_{u}^{\prime}+1\right)\left(2 J_{\ell}+1\right)\left(2 J_{\ell}^{\prime}+1\right) \\
& \quad \times\left\{\begin{array}{ccc}
L_{u} & L_{\ell} & 1 \\
J_{\ell} & J_{u} & S
\end{array}\right\}\left\{\begin{array}{ccc}
L_{u} & L_{\ell} & 1 \\
J_{\ell} & J_{u}^{\prime} & S
\end{array}\right\}\left\{\begin{array}{ccc}
L_{u} & L_{\ell} & 1 \\
J_{\ell}^{\prime} & J_{u} & S
\end{array}\right\} \\
& \quad \times\left\{\begin{array}{ccc}
L_{u} & L_{\ell} & 1 \\
J_{\ell}^{\prime} & J_{u}^{\prime} & S
\end{array}\right\}\left\{\begin{array}{ccc}
K & J_{u}^{\prime} & J_{u} \\
J_{\ell} & 1 & 1
\end{array}\right\}\left\{\begin{array}{ccc}
K & J_{u}^{\prime} & J_{u} \\
J_{\ell}^{\prime} & 1 & 1
\end{array}\right\}\left[P^{(K)}\left(\boldsymbol{\Omega}^{\prime}, \boldsymbol{\Omega}\right)\right]_{i j} \\
& \quad \times \frac{1}{2} \frac{\Phi\left(v_{J_{u} J_{\ell}}-v+\frac{v_{0}}{c} \boldsymbol{v} \cdot \boldsymbol{\Omega}\right)+\Phi\left(v_{J_{u}^{\prime} J_{\ell}}-v+\frac{v_{0}}{c} \boldsymbol{v} \cdot \boldsymbol{\Omega}\right)^{*}}{1+\epsilon^{\prime}+2 \pi \mathrm{i} v_{J_{u}^{\prime} J_{u}} / A\left(L_{u} \rightarrow L_{\ell}\right)} \\
& \quad \times \delta\left(v-\frac{v_{0}}{c} \boldsymbol{v} \cdot \boldsymbol{\Omega}-v^{\prime}+\frac{v_{0}}{c} \boldsymbol{v} \cdot \boldsymbol{\Omega}^{\prime}-v_{J_{\ell}^{\prime} J_{\ell}}\right) .
\end{aligned}
$$

From now on we focus attention on the last terms in the righthand side of Eq. (A.3), which contain the frequency dependence 
of the redistribution matrix:

$$
\begin{aligned}
\mathcal{R}_{\mathrm{II}}(\boldsymbol{v})= & f\left(v-\frac{v_{0}}{c} \boldsymbol{v} \cdot \boldsymbol{\Omega}\right) \\
& \times \delta\left(v-\frac{v_{0}}{c} \boldsymbol{v} \cdot \boldsymbol{\Omega}-v^{\prime}+\frac{v_{0}}{c} \boldsymbol{v} \cdot \boldsymbol{\Omega}^{\prime}-v_{J_{\ell}^{\prime} J_{\ell}}\right),
\end{aligned}
$$

where, in order to simplify the notation, we have not explicitly indicated the dependence of $\mathcal{R}_{\mathrm{II}}(\boldsymbol{v})$ on the various quantities, and where we have set

$$
\begin{aligned}
& \frac{1}{2} \frac{\Phi\left(v_{J_{u} J_{\ell}}-v+\frac{v_{0}}{c} \boldsymbol{v} \cdot \boldsymbol{\Omega}\right)+\Phi\left(v_{J_{u}^{\prime} J_{\ell}}-v+\frac{v_{0}}{c} \boldsymbol{v} \cdot \boldsymbol{\Omega}\right)^{*}}{1+\epsilon^{\prime}+2 \pi \mathrm{i} v_{J_{u}^{\prime} J_{u}} / A\left(L_{u} \rightarrow L_{\ell}\right)} \equiv \\
& f\left(v-\frac{v_{0}}{c} \boldsymbol{v} \cdot \boldsymbol{\Omega}\right) .
\end{aligned}
$$

In order to find the $R_{\mathrm{II}}$ redistribution matrix in the observer's frame, we have to average the quantity $\mathcal{R}_{\mathrm{II}}(\boldsymbol{v})$ over the velocity distribution of the atoms. We assume that the atoms have a Maxwellian velocity distribution characterized by the temperature $T$,

$\mathcal{P}(\boldsymbol{v})=\left(\frac{m}{2 \pi k_{\mathrm{B}} T}\right)^{3 / 2} \exp \left(-\frac{m v^{2}}{2 k_{\mathrm{B}} T}\right)$

with $k_{\mathrm{B}}$ the Boltzmann constant, and $m$ the mass of the atom. We consider a Cartesian reference system defined so that the unit vectors $\boldsymbol{n}_{1}$ and $\boldsymbol{n}_{2}$ lie in the plane defined by $\boldsymbol{\Omega}^{\prime}$ and $\boldsymbol{\Omega}$, and $\boldsymbol{n}_{1}$ bisects the angle $\Theta$ formed by $\boldsymbol{\Omega}^{\prime}$ and $\boldsymbol{\Omega}$. Introducing the dimensionless quantity

$\boldsymbol{u}=\left(\frac{m}{2 k_{\mathrm{B}} T}\right)^{1 / 2} \boldsymbol{v}$,

and the Doppler width

$w=\frac{v_{0}}{c}\left(\frac{2 k_{\mathrm{B}} T}{m}\right)^{1 / 2}$

we have

$\frac{v_{0}}{c} \boldsymbol{v} \cdot \boldsymbol{\Omega}^{\prime}=w\left(\alpha u_{1}+\beta u_{2}\right)$
$\frac{v_{0}}{c} \boldsymbol{v} \cdot \mathbf{\Omega}=w\left(\alpha u_{1}-\beta u_{2}\right)$

with

$\alpha=\cos \frac{\Theta}{2}$, and $\beta=\sin \frac{\Theta}{2}$.

We thus have to calculate

$$
\begin{aligned}
\left\langle\mathcal{R}_{\mathrm{II}}(\boldsymbol{v})\right\rangle= & \int \mathrm{d}^{3} \boldsymbol{v} \mathcal{P}(\boldsymbol{v}) \mathcal{R}_{\mathrm{II}}(\boldsymbol{v}) \\
= & \frac{1}{\pi^{3 / 2}} \int \mathrm{d} u_{1} \int \mathrm{d} u_{2} \int \mathrm{d} u_{3} \mathrm{e}^{-\left(u_{1}^{2}+u_{2}^{2}+u_{3}^{2}\right)} \\
& \times f\left(v-w\left(\alpha u_{1}-\beta u_{2}\right)\right) \delta\left(v-v^{\prime}+2 w \beta u_{2}-v_{J_{\ell}^{\prime} J_{\ell}}\right) .
\end{aligned}
$$

The integral in $\mathrm{d} u_{3}$ can be immediately evaluated, while the one in $\mathrm{d} u_{2}$ can be calculated by exploiting the Dirac delta. Once these integrations are performed, we are left with

$$
\begin{aligned}
\left\langle\mathcal{R}_{\mathrm{II}}(\boldsymbol{v})\right\rangle= & \frac{1}{2 \pi \omega \beta} \exp \left[-\left(\frac{v^{\prime}-v+v_{J_{\ell}^{\prime} J_{\ell}}}{2 w \beta}\right)^{2}\right] \\
& \times \int \mathrm{d} u \mathrm{e}^{-u^{2}} f\left(\frac{v+v^{\prime}+v_{J_{\ell}^{\prime} J_{\ell}}}{2}-w \alpha u\right) .
\end{aligned}
$$

Recalling Eq. (A.5), and recalling the definition of the complex profile $\Phi$ (see Eqs. (15)-(17)), the problem is reduced to the evaluation of integrals of the form

$$
\int \mathrm{d} u \mathrm{e}^{-u^{2}} \frac{1}{\pi} \frac{\Gamma / 4 \pi}{\left(v_{J_{u} J_{\ell}}-\left(v+v^{\prime}+v_{J_{\ell}^{\prime} J_{\ell}}\right) / 2+w \alpha u\right)^{2}+(\Gamma / 4 \pi)^{2}},
$$

and

$$
\int \mathrm{d} u \mathrm{e}^{-u^{2}} \frac{1}{\pi} \frac{\left(v_{J_{u} J_{\ell}}-\left(v+v^{\prime}+v_{J_{\ell}^{\prime} J_{\ell}}\right) / 2+w \alpha u\right)}{\left(v_{J_{u} J_{\ell}}-\left(v+v^{\prime}+v_{J_{\ell}^{\prime} J_{\ell}}\right) / 2+w \alpha u\right)^{2}+(\Gamma / 4 \pi)^{2}} .
$$

Introducing the damping parameter $a=\Gamma / 4 \pi \omega$ and the reduced frequencies

$x_{J_{u} J_{\ell}}=\frac{v_{J_{u} J_{\ell}}-v}{w}$, and $x_{J_{u} J_{\ell}}^{\prime}=\frac{v_{J_{u} J_{\ell}}-v^{\prime}}{w}$,

the first integral can be written as

$$
\begin{aligned}
& \int \mathrm{d} u \mathrm{e}^{-u^{2}} \frac{1}{\alpha w} \frac{1}{\pi} \frac{a / \alpha}{\left(\left(x_{J_{u} J_{\ell}}+x_{J_{u} J_{\ell}^{\prime}}^{\prime}\right) / 2 \alpha+u\right)^{2}+(a / \alpha)^{2}}= \\
& \frac{1}{\alpha w} H\left(\frac{a}{\alpha}, \frac{x_{J_{u} J_{\ell}}+x_{J_{u} J_{\ell}^{\prime}}^{\prime}}{2 \alpha}\right),
\end{aligned}
$$

with $H$ the Voigt function. Similarly, the second integral is given by

$$
\begin{aligned}
& \int \mathrm{d} u \mathrm{e}^{-u^{2}} \frac{1}{\alpha w} \frac{1}{\pi} \frac{\left(x_{J_{u} J_{\ell}}+x_{J_{u} J_{\ell}^{\prime}}^{\prime}\right) / 2 \alpha+u}{\left(\left(x_{J_{u} J_{\ell}}+x_{J_{u} J_{\ell}^{\prime}}^{\prime}\right) / 2 \alpha+u\right)^{2}+(a / \alpha)^{2}}= \\
& \frac{1}{\alpha w} L\left(\frac{a}{\alpha}, \frac{x_{J_{u} J_{\ell}}+x_{J_{u} J_{\ell}^{\prime}}^{\prime}}{2 \alpha}\right),
\end{aligned}
$$

with $L$ the Faraday-Voigt function. Defining the function

$W(a, x)=H(a, x)+\mathrm{i} L(a, x)$,

and observing that $2 \alpha \beta=\sin \Theta$, we find

$$
\begin{aligned}
\left\langle\mathcal{R}_{\mathrm{II}}(\boldsymbol{v})\right\rangle= & \frac{1}{\pi w^{2} \sin \Theta} \exp \left[-\left(\frac{v^{\prime}-v+v_{J_{\ell}^{\prime} J_{\ell}}}{2 w \sin (\Theta / 2)}\right)^{2}\right] \\
& \times \frac{1}{1+\epsilon^{\prime}+2 \pi \mathrm{i}_{J_{u}^{\prime} J_{u}} / A\left(L_{u} \rightarrow L_{\ell}\right)} \\
& \times \frac{1}{2}\left[W\left(\frac{a}{\cos (\Theta / 2)}, \frac{x_{J_{u} J_{\ell}}+x_{J_{u} J_{\ell}^{\prime}}^{2}}{2 \cos (\Theta / 2)}\right)\right. \\
& \left.+W\left(\frac{a}{\cos (\Theta / 2)}, \frac{x_{J_{u}^{\prime} J_{\ell}}+x_{J_{u}^{\prime} J_{\ell}^{\prime}}^{2}}{2 \cos (\Theta / 2)}\right)^{*}\right] .
\end{aligned}
$$

The $R_{\mathrm{II}}$ redistribution matrix in the observer's frame is obtained by substituting the last two lines in the righthand side of Eq. (A.3) with $\left\langle\mathcal{R}_{\mathrm{II}}(\boldsymbol{v})\right\rangle$ (see Eq. (32)). 


\section{Appendix B: Analytical expression of the angle-averaged $\boldsymbol{R}_{\text {|I-AA }}$ redistribution matrix}

We start from the expression of $\mathcal{R}_{\mathrm{II}}(\boldsymbol{v})$ given by Eq. (A.4). Following Hummer (1962), the angle-averaged redistribution matrix is calculated in two steps: first we fix the value of the velocity $\boldsymbol{v}$ and we average the quantity $\mathcal{R}_{\mathrm{II}}(\boldsymbol{v})$ over all possible propagation directions of the incoming and outgoing photons, then we average the ensuing expression over the velocity distribution of the atoms.

We introduce the quantities $\boldsymbol{u}$ and $w$ defined in Eqs. (A.7) and (A.8), and we consider a Cartesian reference system with the $z$-axis directed along the velocity of the atom. Indicating with $\theta^{\prime}$ and $\theta$ the polar angles of the incoming and outgoing photons, respectively, in this reference frame, we have

$$
\begin{aligned}
& \frac{v_{0}}{c} \boldsymbol{v} \cdot \boldsymbol{\Omega}^{\prime}=w u \mu^{\prime}, \\
& \frac{v_{0}}{c} \boldsymbol{v} \cdot \boldsymbol{\Omega}=w u \mu,
\end{aligned}
$$

with $\mu^{\prime}=\cos \theta^{\prime}$ and $\mu=\cos \theta$. The first step is thus to calculate

$$
\begin{aligned}
\mathcal{R}_{\mathrm{II}-\mathrm{AA}}(u)= & \frac{1}{16 \pi^{2}} \oint \mathrm{d} \boldsymbol{\Omega}^{\prime} \oint \mathrm{d} \boldsymbol{\Omega} \mathcal{R}_{\mathrm{II}}(\boldsymbol{v}) \\
= & \frac{1}{4} \int_{-1}^{+1} \mathrm{~d} \mu f(v-w u \mu) \\
& \times \int_{-1}^{+1} \mathrm{~d} \mu^{\prime} \delta\left(v-v^{\prime}-w u\left(\mu-\mu^{\prime}\right)-v_{J_{\ell}^{\prime} J_{\ell}}\right) .
\end{aligned}
$$

Since the integration interval over $\mu^{\prime}$ and $\mu$ is $[-1,1]$, for given values of $\left(v-v^{\prime}\right)$ the singularity of the Dirac delta may fall outside this integration interval, and $\mathcal{R}_{\mathrm{II}-\mathrm{AA}}=0$. Let us therefore focus attention on the integral

$$
\begin{aligned}
I & =\int_{-1}^{+1} \mathrm{~d} \mu^{\prime} \delta\left(v-v^{\prime}-w u\left(\mu-\mu^{\prime}\right)-v_{J_{\ell}^{\prime} J_{\ell}}\right) \\
& =\frac{1}{w u} \int_{-w u}^{+w u} \mathrm{~d} y \delta\left(y-\left(v^{\prime}-v+w u \mu+v_{J_{\ell}^{\prime} J_{\ell}}\right)\right),
\end{aligned}
$$

where we have made the variable change $y=w u \mu^{\prime}$. We thus have

$I=\frac{1}{w u} \quad$ if $\quad-w u \leq v^{\prime}-v+w u \mu+v_{J_{\ell}^{\prime} J_{\ell}} \leq w u$,

$I=0 \quad$ otherwise.

Defining a function $\Lambda(x)$ which is equal to unity if $-1 \leq x \leq 1$, and zero otherwise, we can write

$$
\begin{aligned}
\mathcal{R}_{\mathrm{II}-\mathrm{AA}}(u)= & \frac{1}{4 w u} \int_{-1}^{+1} \mathrm{~d} \mu f(v-w u \mu) \\
& \times \Lambda\left[\mu+\frac{\left(v^{\prime}-v+v_{J_{\ell}^{\prime} J_{\ell}}\right)}{w u}\right],
\end{aligned}
$$

This expression shows that if $u$ is sufficiently small, then the quantity $\left(v^{\prime}-v+v_{J_{f}^{\prime} J_{f}}\right) / w u>1$ and $\Lambda$ is zero for any value of $\mu$. Physically, this means that there is a maximum shift in frequency that can be produced by atoms moving with a given velocity. Let us define

$$
\begin{aligned}
& \bar{v}=\max \left(v, v^{\prime}+v_{J_{\ell}^{\prime} J_{\ell}}\right), \\
& \underline{v}=\min \left(v, v^{\prime}+v_{J_{\ell}^{\prime} J_{\ell}}\right) .
\end{aligned}
$$

If $v<v^{\prime}+v_{J_{\ell}^{\prime} J_{\ell}}$, then in order for the argument of the function $\Lambda$ to fall in the interval $[-1,1]$ (for at least one value of $\mu$ ), it must be

$-1+\frac{v^{\prime}-v+v_{J_{\ell}^{\prime} J_{\ell}}}{w u}=-1+\frac{\bar{v}-\underline{v}}{w u} \leq 1$,

which means that the velocity of the atom must be higher than

$u_{\min }=\frac{\bar{v}-\underline{v}}{2 w}=\frac{\left|v^{\prime}-v+v_{J_{\ell}^{\prime} J_{\ell}}\right|}{2 w}$

It can be easily proven that the same relation is obtained in the case in which $v>v^{\prime}+v_{J_{\ell}^{\prime} J_{\ell}}$, so that Eq. (B.11) is general.

Let us assume again $v<v^{\prime}+v_{J_{\ell}^{\prime} J_{f}}$ (so that $\bar{v}=v^{\prime}+v_{J_{\ell}^{\prime} J_{\ell}}$, and $\underline{v}=v)$. In order for the argument of the function $\Lambda$ to fall in the interval $[-1,1]$, it must be

$-1 \leq \mu \leq 1-\frac{v^{\prime}-v+v_{J_{\ell}^{\prime} J_{\ell}}}{w u}=1-\frac{\bar{v}-\underline{v}}{w u}$,

or, equivalently,

$\bar{v}-w u \leq v-w u \mu \leq \underline{v}+w u$.

Also in this case it can be proven that the same relation is obtained in the case in which $v>v^{\prime}+v_{J_{\ell}^{\prime} J_{\ell}}$, so that Eq. (B.13) is general. Introducing the Heaviside function $F\left(x, x_{0}\right)$, defined so that $F\left(x, x_{0}\right)=1$ for $x \geq x_{0}$, and $F\left(x, x_{0}\right)=0$ for $x<x_{0}$, we have

$\mathcal{R}_{\mathrm{II}-\mathrm{AA}}(u)=\frac{1}{4 w^{2} u^{2}} F\left(u, u_{\min }\right) \int_{\bar{v}-w u}^{\underline{v}+w u} f(y) \mathrm{d} y$,

with $y=v-w u \mu$.

The next step is to average $\mathcal{R}_{\mathrm{II}-\mathrm{AA}}(u)$ over the velocity distribution (that we assume to be Maxwellian)

$\mathcal{P}(u)=\frac{1}{\pi^{3 / 2}} \mathrm{e}^{-u^{2}} 4 \pi u^{2}$.

We thus have to calculate

$$
\begin{aligned}
\left\langle\mathcal{R}_{\mathrm{II}-\mathrm{AA}}(u)\right\rangle & =\int \mathrm{d} u \mathcal{P}(u) \mathcal{R}_{\mathrm{II}-\mathrm{AA}}(u) \\
& =\frac{1}{w^{2} \sqrt{\pi}} \int_{u_{\min }}^{\infty} \mathrm{d} u \mathrm{e}^{-u^{2}} \int_{\bar{v}-w u}^{\underline{v}+w u} f(y) \mathrm{d} y .
\end{aligned}
$$

Recalling Eq. (A.5), and the definition of the complex profile $\Phi$ (see Eqs. (15), (16), and (17)), the problem is reduced to the evaluation of the following integrals

$$
\begin{array}{r}
\int_{\bar{v}-w u}^{\underline{v}+w u} \frac{\Gamma^{\prime}}{\Gamma^{\prime 2}+\left(v_{0}-y\right)^{2}} \mathrm{~d} y=\int_{\left(\bar{v}-v_{0}-w u\right) / \Gamma^{\prime}}^{\left(\underline{\nu}-v_{0}+w u\right) / \Gamma^{\prime}} \frac{\mathrm{d} z}{1+z^{2}}= \\
\tan ^{-1}\left(\frac{\underline{v}-v_{0}+w u}{\Gamma^{\prime}}\right)-\tan ^{-1}\left(\frac{\bar{v}-v_{0}-w u}{\Gamma^{\prime}}\right)
\end{array}
$$

and

$$
\begin{aligned}
\int_{\bar{v}-w u}^{\underline{v}+w u} \frac{v_{0}-y}{\Gamma^{\prime 2}+\left(v_{0}-y\right)^{2}} \mathrm{~d} y=-\int_{\left(\bar{v}-v_{0}-w u\right) / \Gamma^{\prime}}^{\left(\underline{\nu}-v_{0}+w u\right) / \Gamma^{\prime}} \frac{z}{1+z^{2}} \mathrm{~d} z= \\
\frac{1}{2}\left[\ln \left(1+\left(\frac{\bar{v}-v_{0}-w u}{\Gamma^{\prime}}\right)^{2}\right)-\ln \left(1+\left(\frac{\underline{v}-v_{0}+w u}{\Gamma^{\prime}}\right)^{2}\right)\right],
\end{aligned}
$$

where $\Gamma^{\prime}=\Gamma / 4 \pi$, and where, in both cases, we have made the variable change $z=\left(y-v_{0}\right) / \Gamma^{\prime}$. Introducing the function

$h(x)=\tan ^{-1}(x)-\frac{\mathrm{i}}{2} \ln \left(1+x^{2}\right)$, 
L. Belluzzi and J. Trujillo Bueno: The transfer of resonance line polarization with PRD and $J$-state interference
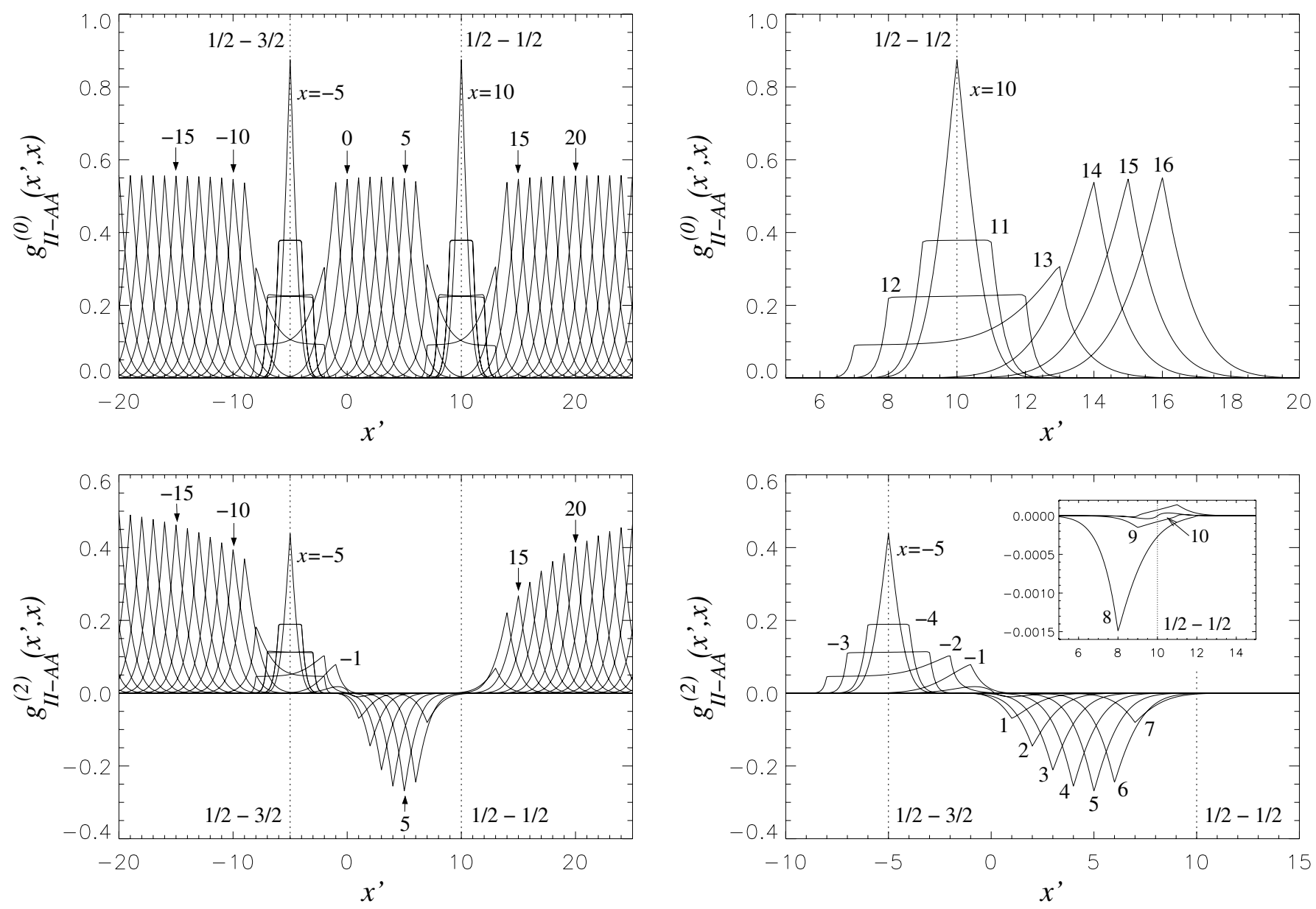

Fig. C.1. Upper left: plot of the $g_{\mathrm{II}-\mathrm{AA}}^{(0)}$ redistribution function defined in Eq. (61), as a function of the reduced frequency of the incoming photons $\left(x^{\prime}\right)$. Each curve corresponds to a different (integer) value of the reduced frequency of the outgoing photon $(x)$. For some curves, the value of $x$ is indicated in the plot. The reduced frequencies $x$ and $x^{\prime}$ are defined with respect to the frequency $v_{0}$ corresponding to the energy separation between the centers of gravity of the two terms $\left(x=\left(v_{0}-v\right) / \Delta v_{\mathrm{D}}, x^{\prime}=\left(v_{0}-v^{\prime}\right) / \Delta v_{\mathrm{D}}\right)$. We consider a ${ }^{2} S-{ }^{2} P$ transition, the two fine-structure components having a separation of 15 Doppler widths (the energies of the upper levels are calculated according to the $L-S$ coupling scheme). We assume an Einstein coefficient $A_{u \ell}=10^{8} \mathrm{~s}^{-1}$, a Doppler width $\Delta \lambda_{\mathrm{D}}=60 \mathrm{~m} \AA$, and we accordingly calculate the damping constant as $a=A_{u \ell} /\left(4 \pi \Delta v_{\mathrm{D}}\right) \approx 10^{-3}$ (collisional broadening is neglected, and the conversion of the Doppler width from wavelength units to frequency units is made assuming that the transitions fall at $5000 \AA$ ). Upper right: same as upper left, but considering only a few values of $x$ (indicated in the plot). Lower left: same as upper left, but for the $g_{\mathrm{II}-\mathrm{AA}}^{(2)}$ redistribution function. Lower right: same as lower left, but considering only a few values of $x$ (indicated in the plot). The inset panel is a zoom of the line core region of the $1 / 2-1 / 2$ transition, showing in more details the curves obtained for values of $x$ close to $x=10$ (the frequency position of the $1 / 2-1 / 2$ transition).

and defining the quantities

$$
\begin{aligned}
& \underline{x}_{J_{a} J_{b}, J_{c}}=\frac{\underline{v}-v_{J_{a} J_{b}}}{w}, \text { with } \quad \underline{v}=\min \left(v, v^{\prime}+v_{J_{c} J_{b}}\right), \\
& \bar{x}_{J_{a} J_{b}, J_{c}}=\frac{\bar{v}-v_{J_{a} J_{b}}}{w}, \text { with } \quad \bar{v}=\max \left(v, v^{\prime}+v_{J_{c} J_{b}}\right),
\end{aligned}
$$

we find:

$$
\begin{aligned}
\left\langle\mathcal{R}_{\mathrm{II}-\mathrm{AA}}(u)\right\rangle= & \frac{1}{1+\epsilon^{\prime}+2 \pi \mathrm{i} v_{J_{u}^{\prime} J_{u}} / A\left(L_{u} \rightarrow L_{\ell}\right)} \\
\times & \frac{1}{\pi^{3 / 2} w^{2}} \int_{u_{\min }}^{\infty} \mathrm{d} u \mathrm{e}^{-u^{2}} \\
\times & \frac{1}{2}\left\{\left[h\left(\frac{\underline{x}_{J_{u} J_{\ell}, J_{\ell}^{\prime}}+u}{a}\right)-h\left(\frac{\bar{x}_{J_{u} J_{\ell}, J_{\ell}^{\prime}}-u}{a}\right)\right]\right. \\
& \left.\quad+\left[h\left(\frac{\underline{x}_{J_{u}^{\prime} J_{\ell}, J_{\ell}^{\prime}}+u}{a}\right)-h\left(\frac{\bar{x}_{J_{u}^{\prime} J_{\ell}, J_{\ell}^{\prime}}-u}{a}\right)\right]^{*}\right\},
\end{aligned}
$$

with $a=\Gamma^{\prime} / w=\Gamma / 4 \pi w$ the damping constant. The angle averaged $R_{\mathrm{II}-\mathrm{AA}}\left(v^{\prime}, \boldsymbol{\Omega}^{\prime} ; v, \boldsymbol{\Omega}\right)$ redistribution matrix is then obtained by substituting the last two lines in the righthand side of Eq. (A.3) with the above expression of $\left\langle\mathcal{R}_{\mathrm{II}-\mathrm{AA}}(u)\right\rangle$.

\section{Appendix C: Analysis of the functions $g_{\|-A A}^{(K)}$}

The redistribution functions $g_{\mathrm{II}-\mathrm{AA}}^{(K)}$ (see Eq. (61)) for a ${ }^{2} S-{ }^{2} P$ transition are plotted in Fig. C.1 as a function of the reduced frequency of the incoming photon $\left(x^{\prime}\right)$, and for different values of the reduced frequency of the outgoing photon $(x)$. It can be immediately observed that the redistribution functions $g_{\mathrm{II}-\mathrm{AA}}^{(0)} \mathrm{co}-$ incide with those obtained in the unpolarized case (cf. Jefferies $\&$ White 1960). These functions show that scattering is strongly non-coherent in the core of the line, where the complete redistribution is actually a good approximation, and nearly coherent in the wings. As known, the most critical region, where the redistribution functions are sensibly asymmetric and PRD effects are 
more important, is between about 2 and 4 Doppler widths from the line center (see the upper right panel of Fig. C.1).

For values of $x$ in the core of the 1/2-3/2 transition, the redistribution functions $g_{\mathrm{II}-\mathrm{AA}}^{(2)}$ are very similar (except for a scaling factor) to $g_{\mathrm{II}-\mathrm{AA}}^{(0)}$. Due to the $J$-state interference terms, such redistribution functions become negative between the two lines, while they practically vanish for values of $x$ in the core of the $1 / 2-1 / 2$ transition (we recall that the polarizability factor of this transition is zero). It can be observed that for $x=10$ (i.e., for an outgoing photon at the line-center of the $1 / 2-1 / 2$ transition), the $g_{\mathrm{II}-\mathrm{AA}}^{(2)}$ shows an antisymmetric shape that vanishes for $x^{\prime}=10$ (see the inner plot in the lower right panel of Fig. C.1). The amplitude of this redistribution function for $x=10$ is, however, extremely small for any value of $x^{\prime}$.

\section{Appendix D: Calculation of the scattering integral}

Let $v_{i}$, with $i=1, \ldots, N_{\mathrm{f}}$ be the frequency grid of the problem. At each height of the atmospheric model, and for each frequency $v_{i}$ of the scattered photon, we have to calculate the integral

$\hat{J}_{Q}^{K}\left(v_{i}\right)=\int \mathrm{d} v^{\prime} J_{Q}^{K}\left(v^{\prime}\right) g_{\mathrm{II}-\mathrm{AA}}^{(K)}\left(v^{\prime}, v_{i}\right)$.

We want to calculate this integral by introducing a suitable quadrature rule. It is well known that a quadrature scheme works well when the integrand varies slowly and without discontinuities, so that it is sufficient to consider a limited number of points. The difficulty in evaluating the integral of Eq. (D.1) is due to the fact that while the function $J_{Q}^{K}\left(v^{\prime}\right)$ varies slowly outside the line core regions, the functions $g_{\mathrm{II}-\mathrm{AA}}^{(K)}\left(v^{\prime}, v_{i}\right)$ are sharply peaked at $v^{\prime}=v_{i}$ (where they have a discontinuity in slope), and they are different from zero only within a small frequency interval (a few Doppler widths) centered at $v_{i}$. Since in our problem we have to consider quite large frequency intervals, spanning whole multiplets, an impractically large number of frequency points would be necessary to cover such intervals with a spacing smaller than one Doppler width. We thus calculate this integral following the quadrature scheme based on the cardinal natural cubic splines functions, proposed by Adams et al. (1971). This quadrature scheme is based on the idea to describe $J_{Q}^{K}\left(v^{\prime}\right)$ through a simple function, and to take the behavior of $g_{\mathrm{II}-\mathrm{AA}}^{(K)}\left(v^{\prime}, v_{i}\right)$ into account more precisely.

Let $\psi_{j}(v), j=1, \ldots, N_{\mathrm{f}}$ be the set of cardinal natural cubic splines corresponding to the frequency grid of the problem. These splines functions satisfy the requirement

$\psi_{j}\left(v_{k}\right)=\delta_{j k}$

(see Ahlberg et al. 1967; Adams et al. 1971; Stoer \& Bulirsch 1980 , for a discussion of the basic properties of splines). The spline representation of $J_{Q}^{K}\left(v^{\prime}\right)$ is given by

$\mathcal{J}_{Q}^{K}\left(v^{\prime}\right)=\sum_{j=1}^{N_{\mathrm{f}}} J_{Q}^{K}\left(v_{j}\right) \psi_{j}\left(v^{\prime}\right)$.

Replacing $J_{Q}^{K}\left(v^{\prime}\right)$ by its spline representation in the scattering integral, we have

$$
\begin{aligned}
\hat{J}_{Q}^{K}\left(v_{i}\right) & =\int \mathrm{d} v^{\prime} \sum_{j=1}^{N_{\mathrm{f}}} J_{Q}^{K}\left(v_{j}\right) \psi_{j}\left(v^{\prime}\right) g_{\mathrm{II}-\mathrm{AA}}^{(K)}\left(v^{\prime}, v_{i}\right) \\
& =\sum_{j=1}^{N_{\mathrm{f}}} J_{Q}^{K}\left(v_{j}\right) w_{i j}^{(K)} .
\end{aligned}
$$

The problem is thus shifted to the calculation of the weights

$w_{i j}^{(K)}=\int \mathrm{d} v^{\prime} \psi_{j}\left(v^{\prime}\right) g_{\mathrm{II}-\mathrm{AA}}^{(K)}\left(v^{\prime}, v_{i}\right)$

This integral can be calculated through the usual quadrature techniques (e.g., the trapezoidal rule) taking into account that $g_{\mathrm{II}-\mathrm{AA}}^{(K)}\left(v^{\prime}, v_{i}\right)$ becomes very small after a few Doppler widths from $v_{i}$, and that it has a discontinuity in slope in $v^{\prime}=v_{i}$. A very fine frequency grid is thus generally needed for the evaluation of the integral appearing in the definition of the weights $w_{i j}^{(K)}$. However, the weights have to be calculated only once, and the frequency grid of the problem, which is used for the calculation of the scattering integral through the quadrature rule of Eq. (D.4), can be chosen with an appropriate and manageable number of points.

Particular attention has to be paid at the boundaries of the frequency grid, since the natural cubic splines $\psi_{j}(v)$ are not defined for $v<v_{1}$ and $v>v_{N_{\mathrm{f}}}$ (we assume that the frequency grid is monotonically increasing, so that $v_{1}$ is the lowest frequency and $v_{N_{\mathrm{f}}}$ is the highest one). Following Uitenbroek (1989), we overcome this problem by choosing the outermost grid points sufficiently far from the multiplet that any frequency redistribution effect with other points of the frequency grid can be safely neglected. At the boundary points we thus consider the $R_{\mathrm{II}-\mathrm{AA}}$ redistribution matrix corresponding to the case of purely coherent scattering in the observer's frame (this is indeed a good approximation in the far wings of a spectral line, as shown by Jefferies \& White 1960). Such redistribution matrix is given by Eq. (30) (which describes purely coherent scattering in the atom rest frame), with the only difference that the functions $\phi$ and $\psi$ appearing in the complex profile $\Phi$ are now the Voigt and Faraday-Voigt profiles, respectively. Thanks to the presence of the factor $\delta\left(v-v^{\prime}-v_{J_{f}^{\prime} J_{f}}\right)$, which guarantees the coherency of scattering, the evaluation of the scattering integral for this redistribution matrix does not present any difficulty.

It should be observed that for the two-level atom case, the calculation of the scattering integral can be substantially simplified by considering suitable approximations (which depend on the spectral region considered: line-core, wings, "transition" region) of the functions $g_{\mathrm{II}-\mathrm{AA}}^{(K)}$ (see Gouttebroze 1986; Uitenbroek 1989). Unfortunately, such approximations cannot be easily generalized to the case of a two-term atom, due to the presence of the $J$-state interference terms in the functions $g_{\mathrm{II}-\mathrm{AA}}^{(K)}$.

The evaluation of the integral

$\bar{J}_{Q}^{K}=\int \mathrm{d} v^{\prime} J_{Q}^{K}\left(v^{\prime}\right) \varphi\left(v^{\prime}\right)$,

does not present particular difficulties, and can be performed with a trapezoidal quadrature, provided that the frequency grid $v_{i}, i=1, \ldots, N_{\mathrm{f}}$ is sufficiently fine in the core of the lines. We thus have

$\bar{J}_{Q}^{K}=\sum_{j=1}^{N_{\mathrm{f}}} J_{Q}^{K}\left(v_{j}\right) \varphi\left(v_{j}\right) u_{j}$,

with the weights $u_{j}$ given by

$$
\begin{aligned}
u_{1} & =\frac{v_{2}-v_{1}}{2}, \\
u_{j} & =\frac{v_{j+1}-v_{j-1}}{2}, \quad j=2, \ldots, N_{\mathrm{f}}-1, \\
u_{N_{\mathrm{f}}} & =\frac{v_{N_{\mathrm{f}}}-v_{N_{\mathrm{f}}-1}}{2} .
\end{aligned}
$$


We conclude with a discussion of the normalization of the weights $w_{i j}^{(K)}$ and $u_{j}$ appearing in Eqs. (D.5) and (D.8), respectively. One requirement that must be satisfied is that in LTE conditions, when $J_{Q}^{K}(v)=B_{T}(v) \delta_{K 0} \delta_{Q 0}$, the Kirchhoff law, $\varepsilon_{I}(v, \boldsymbol{\Omega}) / \eta_{I}(v)=B_{T}(v)$ must be recovered. Recalling Eqs. (53) and (55), this implies

$$
\int \mathrm{d} v^{\prime}\left[\alpha r_{\mathrm{II}-\mathrm{AA}}^{(0)}\left(v^{\prime}, v_{i}\right)+(1-\alpha) r_{\mathrm{III}}^{(0)}\left(v^{\prime}, v_{i}\right)\right]=\frac{1}{1+\epsilon^{\prime}} \varphi\left(v_{i}\right)
$$

or, equivalently, recalling that $r_{\mathrm{III}}^{(0)}\left(v^{\prime}, v_{i}\right)=\varphi\left(v^{\prime}\right) \varphi\left(v_{i}\right) /\left(1+\epsilon^{\prime}\right)$,

$\int \mathrm{d} v^{\prime}\left[\alpha g_{\mathrm{II}-\mathrm{AA}}^{(0)}\left(v^{\prime}, v_{i}\right)+(1-\alpha) \frac{\varphi\left(v^{\prime}\right)}{1+\epsilon^{\prime}}\right]=\frac{1}{1+\epsilon^{\prime}}$,

Applying the quadrature schemes previously discussed, we must have

$$
\begin{aligned}
\int \mathrm{d} v^{\prime} g_{\mathrm{II}-\mathrm{AA}}^{(0)}\left(v^{\prime}, v_{i}\right) & =\int \mathrm{d} v^{\prime} g_{\mathrm{II}-\mathrm{AA}}^{(0)}\left(v^{\prime}, v_{i}\right) \sum_{j=1}^{N_{\mathrm{f}}} \psi_{j}\left(v^{\prime}\right) \\
& =\sum_{j=1}^{N_{\mathrm{f}}} \int \mathrm{d} v^{\prime} g_{\mathrm{II}-\mathrm{AA}}^{(0)}\left(v^{\prime}, v_{i}\right) \psi_{j}\left(v^{\prime}\right) \\
& =\sum_{j=1}^{N_{\mathrm{f}}} w_{i j}^{(0)}=\frac{1}{1+\epsilon^{\prime}},
\end{aligned}
$$

and

$$
\int \mathrm{d} v^{\prime} \varphi\left(v^{\prime}\right)=\sum_{j=1}^{N_{\mathrm{f}}} \varphi\left(v_{j}\right) u_{j}=1
$$

Once the frequency grid has been selected and the weights have been numerically evaluated, we renormalize them so that Eqs. (D.11) and (D.12) are satisfied. For $w_{i j}^{(2)}$ we use the same renormalization as for $w_{i j}^{(0)}$.

\section{Appendix E: Calculation of the new estimate of $S_{0}^{0}$ through the FBF method}

At each height in the atmosphere, we have to calculate the quantities $\Delta S_{0}^{0}\left(v_{i}\right)$ with $i=1, \ldots, N_{\mathrm{f}}$ by solving the system of equations (see Eq. (75))

$\Delta S_{0}^{0}\left(v_{i}\right)=\int \mathrm{d} v^{\prime} g^{(0)}\left(v^{\prime}, v_{i}\right) r_{v^{\prime}} \Lambda_{00,00}\left(v^{\prime}\right) \Delta S_{0}^{0}\left(v^{\prime}\right)+\rho\left(v_{i}\right)$,

where

$\rho\left(v_{i}\right)=\tilde{J}_{0}^{0}\left(v_{i}\right)^{\text {old }}+\frac{\epsilon^{\prime}}{1+\epsilon^{\prime}} B_{T}\left(v_{0}\right)-S_{0}^{0}\left(v_{i}\right)^{\text {old }}$,

and where, with a small modification of the notation that should create no confusion, we have deleted the explicit dependence of the various quantities on the spatial grid point (here fixed).

Substituting the explicit expression of the quantity $g^{(0)}\left(v^{\prime}, v_{i}\right)$ (see Eq. (57)), the integral in the righthand side of Eq. (E.1) is given by

$$
\begin{aligned}
& \alpha \int \mathrm{d} v^{\prime} g_{\mathrm{II}-\mathrm{AA}}^{(0)}\left(v^{\prime}, v_{i}\right) r_{v^{\prime}} \Lambda_{00,00}\left(v^{\prime}\right) \Delta S_{0}^{0}\left(v^{\prime}\right) \\
& +(1-\alpha) \int \mathrm{d} v^{\prime} \frac{\varphi\left(v^{\prime}\right)}{1+\epsilon^{\prime}} r_{v^{\prime}} \Lambda_{00,00}\left(v^{\prime}\right) \Delta S_{0}^{0}\left(v^{\prime}\right) .
\end{aligned}
$$

We calculate these integrals by means of the quadrature rules described in Appendix (D). Considering the spline representation of the quantity $r_{v^{\prime}} \Lambda_{00,00}\left(v^{\prime}\right) \Delta S_{0}^{0}\left(v^{\prime}\right)$ :

$r_{v^{\prime}} \Lambda_{00,00}\left(v^{\prime}\right) \Delta S_{0}^{0}\left(v^{\prime}\right) \approx \sum_{j=1}^{N_{\mathrm{f}}} r_{v_{j}} \Lambda_{00,00}\left(v_{j}\right) \Delta S_{0}^{0}\left(v_{j}\right) \psi_{j}\left(v^{\prime}\right)$,

with $\psi_{j}\left(v^{\prime}\right)$ the cardinal natural cubic splines corresponding to the frequency grid of the problem (see Appendix D), the first integral in Eq. (E.3) is given by

$$
\begin{aligned}
& \alpha \int \mathrm{d} v^{\prime} g_{\mathrm{II}-\mathrm{AA}}^{(0)}\left(v^{\prime}, v_{i}\right) r_{v^{\prime}} \Lambda_{00,00}\left(v^{\prime}\right) \Delta S_{0}^{0}\left(v^{\prime}\right) \\
& =\alpha \sum_{j=1}^{N_{\mathrm{f}}} r_{v_{j}} \Lambda_{00,00}\left(v_{j}\right) \Delta S_{0}^{0}\left(v_{j}\right) \int \mathrm{d} v^{\prime} g_{\mathrm{II}-\mathrm{AA}}^{(0)}\left(v^{\prime}, v_{i}\right) \psi_{j}\left(v^{\prime}\right) \\
& =\alpha \sum_{j=1}^{N_{\mathrm{f}}} r_{v_{j}} \Lambda_{00,00}\left(v_{j}\right) \Delta S_{0}^{0}\left(v_{j}\right) w_{i j}^{(0)},
\end{aligned}
$$

where the weights $w_{i j}^{(0)}$ are defined by Eq. (D.5).

The second integral in Eq. (E.3) can be solved with a trapezoidal quadrature rule:

$(1-\alpha) \int \mathrm{d} v^{\prime} \frac{\varphi\left(v^{\prime}\right)}{1+\epsilon^{\prime}} r_{v^{\prime}} \Lambda_{00,00}\left(v^{\prime}\right) \Delta S_{0}^{0}\left(v^{\prime}\right)=$
$\frac{1-\alpha}{1+\epsilon^{\prime}} \sum_{j=1}^{N_{\mathrm{f}}} r_{v_{j}} \Lambda_{00,00}\left(v_{j}\right) \Delta S_{0}^{0}\left(v_{j}\right) \varphi\left(v_{j}\right) u_{j}$,

where the weights $u_{j}$ are defined by Eq. (D.8).

By applying these quadrature schemes, the initial system of equations takes the form

$$
\begin{aligned}
\Delta S_{0}^{0}\left(v_{i}\right)=\sum_{j=1}^{N_{\mathrm{f}}} & r_{v_{j}} \Lambda_{00,00}\left(v_{j}\right) \Delta S_{0}^{0}\left(v_{j}\right) \\
& \times\left[\alpha w_{i j}^{(0)}+\frac{1-\alpha}{1+\epsilon^{\prime}} \varphi\left(v_{j}\right) u_{j}\right]+\rho\left(v_{i}\right) .
\end{aligned}
$$

Indicating with $\Delta \boldsymbol{S}_{0}^{0}$ and $\boldsymbol{\rho}$ two column vectors whose elements contain the values $\Delta S_{0}^{0}\left(v_{i}\right)$ and of $\rho\left(v_{i}\right)$ at the various frequencies, the system can also be written in the form

$$
\mathbf{M} \Delta S_{0}^{0}=\rho,
$$

where the elements of the matrix $\mathbf{M}$ are given by

$$
M_{i j}=\delta_{i j}-r_{v_{j}} \Lambda_{00,00}\left(v_{j}\right)\left[\alpha w_{i j}^{(0)}+\frac{1-\alpha}{1+\epsilon^{\prime}} \varphi\left(v_{j}\right) u_{j}\right] .
$$

The values $\Delta S_{0}^{0}\left(v_{i}\right)$ at the various frequencies are then calculated by solving numerically (through matrix inversion techniques) the system (E.8). This numerical scheme for the calculation of the new estimates of $S_{0}^{0}$ is generally referred to as "FrequencyBy-Frequency" method.

\section{References}

Adams, T. F., Hummer, D. G., \& Rybicki, G. B. 1971, J. Quant. Spectr. Rad. Transf., 11, 1365

Ahlberg, J. H., Nilson, E. N., \& Walsh J. L. 1967, The Theory of Splines and Their Applications (New York: Academic Press)

Belluzzi, L., \& Trujillo Bueno, J. 2011, ApJ, 743, 3

Belluzzi, L., \& Trujillo Bueno, J. 2012, ApJ, 750, L11

Belluzzi, L., \& Trujillo Bueno, J. 2013, ApJ, 744, L28 
Belluzzi, L., Trujillo Bueno, J., \& Stepan, J. 2012, ApJ, 755, L2

Belluzzi, L., Landi Degl'Innocenti, E., \& Trujillo Bueno, J. 2013, A\&A, 551, 84

Bommier, V. 1997a, A\&A, 328, 706

Bommier, V. 1997b, A\&A, 328, 726

Casini, R., \& Landi Degl'Innocenti, E. 2007, in Plasma Polarization

Spectroscopy, eds. T. Fujimoto, \& A. Iwamae (Berlin: Springer), 249

Derouich, M., Trujillo Bueno, J., \& Manso Sainz, R. 2007, A\&A, 472, 269

Domke, H., \& Hubeny, I. 1988, ApJ, 334, 527

Fontenla, J. M., Avrett, E. H., \& Loeser, R. 1993, ApJ, 406, 319

Gouttebroze, P. 1986, A\&A, 160, 195

Hubeny, I., Oxenius, J., \& Simonneau, E. 1983a, J. Quant. Spectr. Rad. Transf, 29,477

Hubeny, I., Oxenius, J., \& Simonneau, E. 1983b, J. Quant. Spectr. Rad. Transf, 29,495

Hummer, D. G. 1962, MNRAS, 125, 21

Jefferies, J. T., \& White, O. R. 1960, ApJ, 132, 767

Kunasz, P., \& Auer, L. H. 1988, J. Quant. Spectr. Rad. Transf, 39, 67

Landi Degl'Innocenti, E. 1983, Sol. Phys., 85, 3

Landi Degl'Innocenti, E. 1984, Sol. Phys., 91, 1

Landi Degl'Innocenti, E. 1998, Nature, 392, L256

Landi Degl'Innocenti, E., \& Landolfi, M. 2004, Polarization in Spectral Lines (Dordrecht: Kluwer)

Landi Degl'Innocenti, E., Landi Degl'Innocenti, M., \& Landolfi, M. 1997, in Science with THÉMIS, eds. N. Mein, \& S. Sahal-Bréchot (Paris: Obs. Paris-Meudon), 59
Mihalas, D. 1978, Stellar Atmospheres, 2nd edn. (San Francisco: W. H. Freeman and Company)

Rees, D. E., \& Saliba G. J. 1982, A\&A, 115, 1

Sahal-Bréchot, S., Vogt, E., Thoraval, S., \& Diedhiou, I. 1996, A\&A, 309, 317

Sahal-Bréchot, S., Derouich, M., Bommier, V., \& Barklem, P. S. 2007, A\&A, 465, 667

Sampoorna, M., \& Trujillo Bueno, J. 2010, ApJ, 712, 1331

Smitha, H. N., Sampoorna, M., Nagendra, K. N., \& Stenflo, J. O. 2011, ApJ, 733,4

Smitha, H. N., Nagendra, K. N., Sampoorna, M., \& Stenflo, J. O. 2013, J. Quant. Spectr. Rad. Transf, 115, 46

Stenflo, J. O. 1980, A\&A, 84, 68

Stenflo, J. O. 1994, Solar Magnetic Fields: Polarized Radiation Diagnostics (Dordrecht: Kluwer)

Stenflo, J. O., \& Keller, C. U. 1997, Nature, 382, 588

Štěpán, J., \& Trujillo Bueno, J. 2011, ApJ, 732, 80

Štěpán, J., \& Trujillo Bueno, J. 2013, A\&A, 557, 143

Stoer, J., \& Bulirsch, R. 1980, Introduction to Numerical Analysis (New York: Springer)

Trujillo Bueno, J., \& Manso Sainz, R. 1999, ApJ, 516, 436

Trujillo Bueno, J., Štěpán, J., \& Belluzzi, L. 2012, ApJ, 746, L9

Uitenbroek, H. 1989, A\&A, 216, 310

Uitenbroek, H. 2001, ApJ, 557, 389 